\title{
Colloquium: Light scattering by particle and hole arrays
}

\author{
F. J. García de Abajo \\ Instituto de Óptica-CSIC, Serrano 121, 28006 Madrid, Spain \\ (Published 10 October 2007)
}

\begin{abstract}
This Colloquium analyzes the interaction of light with two-dimensional periodic arrays of particles and holes. The enhanced optical transmission observed in the latter and the presence of surface modes in patterned metal surfaces is thoroughly discussed. A review of the most significant discoveries in this area is presented first. A simple tutorial model is then formulated to capture the essential physics involved in these phenomena, while allowing analytical derivations that provide deeper insight. Comparison with more elaborated calculations is offered as well. Finally, hole arrays in plasmon-supporting metals are compared to perforated perfect conductors, thus assessing the role of plasmons in these types of structures through analytical considerations. The developments that have been made in nanophotonics areas related to plasmons in nanostructures, extraordinary optical transmission in hole arrays, complete resonant absorption and emission of light, and invisibility in structured metals are illustrated in this Colloquium in a comprehensive, tutorial fashion.
\end{abstract}

DOI: $10.1103 /$ RevModPhys.79.1267

PACS number(s): 42.25.Fx, 73.20.Mf, 42.79.Dj, 41.20.Jb

\section{CONTENTS}

I. Introduction

II. Overview of Existing Results
A. Single holes
B. Optical transmission through hole arrays
C. Particles

III. Tutorial Approach

A. Basic relations

1. Reflection and absorption in particle arrays

2. Narrowing line shapes through dynamical scattering

B. Lattice singularities

C. Hole arrays

1. Babinet's principle and hole arrays in thin screens

2. Single holes in thick films

3. Hole arrays in thick films

D. Lattice surface modes in structured metals

E. Interplay between lattice and site resonances

F. Slit and cylinder arrays

IV. Real Metals vs Perfect Conductors

A. Surface plasmons

B. Polarization schemes

C. Dipole-dipole interaction

D. Discrepancies in lattice resonances and enhanced transmission

V. Conclusion

Acknowledgments

References

\section{INTRODUCTION}

The scattering of waves in periodic media plays a central role in areas of physics as diverse as low-energy electron diffraction (Pendry, 1974) or atomic-beam scattering from crystal surfaces (Farías and Rieder, 1998). Valence electrons in solids, sound in certain ordered constructions (Martínez-Sala et al., 1995), or light in photonic crystals (Joannopoulos et al., 1997; López, 2003) undergo diffraction that under certain conditions can limit their propagation in frequency regions known as band gaps (Ashcroft and Mermin, 1976). Among these examples, the scattering of electromagnetic waves is particularly important because it allows obtaining structural and spectroscopic information over a fantastically wide range of lengths, going from atomic dimensions in x-ray scattering (Henke et al., 1993) to macroscopic distances in radio and microwaves. Actually, Maxwell's equations are written in first-order derivatives with respect to spatial coordinates, so that light scattering in the absence of nonlinear effects is solely controlled by the shape and permittivity of diffracting objects with distances measured in units of the wavelength, and therefore the same phenomena are encountered over entirely different length scales.

We can classify the performance of periodic structures in three distinct categories according to the ratio of the period $a$ to the wavelength $\lambda$. For $\lambda \gg a$, an effective homogeneous medium description is possible. This is in fact what happens in most naturally occurring substances when $a$ has atomic dimensions. But also in certain artificially textured materials (metamaterials), which allow achieving exotic behavior like magnetic response at visible frequencies (Grigorenko et al., 2005) and media with negative refraction index (Smith et al., 2004), without neglecting the exciting possibility of using nanoparticles as building blocks to tailor on-demand optical properties (Liz-Marzán, 2006). The opposite limit $(\lambda$ $\ll a$ ) is generally well accounted for by classical rays, although keeping track of phases proves to be crucial near points of light accumulation, like in the self-imaging of gratings described by Talbot (Talbot, 1836; Huang et al., 2007). Nevertheless, it is the intermediate regime, when $\lambda$ is comparable to $a$, in which diffraction shows up in full display. We find examples of this in both threedimensional (3D) photonic crystals, which offer a prom- 
ising route to fully controlling light propagation over distances comparable to the wavelength (Joannopoulos et al., 1997; López, 2003), and two-dimensional (2D) crystals, in which a substantial degree of optical confinement has been accomplished (Akahane et al., 2003).

In this Colloquium, we focus on light scattering by planar structures of particles or holes, which have become a current subject of intense research driven to some extent by advances in nanopatterning techniques. Our main purpose is to explain the phenomena observed within this context in a tutorial but nevertheless comprehensive fashion. We first review experimental and theoretical developments in Sec. II. Then, we formulate in Sec. III a simple powerful model that deals with the response of particle and hole arrays on a common footing, leading to analytical expressions that capture the main physical aspects of these systems. Finally, metals with plasmons will be discussed, and the main differences with respect to plasmon-free perfect conductors elucidated, in Sec. IV. We use Gaussian units, unless otherwise stated.

The beginning of the last century witnessed important developments in diffraction of light in gratings after Wood's observation of anomalous reflection bands (Wood, 1902, 1912, 1935) and their subsequent interpretation (Lord Rayleigh, 1907; Fano, 1936, 1941). Two types of anomalies were identified, one of them occurring when a diffracted beam becomes grazing to the plane of the grating, the Rayleigh condition (Lord Rayleigh, 1907), giving rise to a sharp bright band, and the other one showing up to the red of the former as an extended feature containing two neighboring dark and bright bands (Fano, 1936, 1941).

The century concluded with another significant discovery (Ebbesen et al., 1998): periodic arrays of subwavelength holes drilled in thin metallic films can transmit much more light per hole at certain frequencies than what was previously expected for single openings, based upon Bethe's prediction of a severe cutoff in transmission as $(b / \lambda)^{4}$ for large $\lambda$ compared to the hole radius $b$ (Bethe, 1944). Previous knowledge gathered by electrical engineers in the microwave domain (Ulrich, 1967; Chen, 1971; McPhedran et al., 1980) had already exploited the use of periodically drilled surfaces as frequency-selective filters and discussed the occurrence of $100 \%$ transmission at wavelengths slightly above the period. However, the hole sizes that were considered in that context lie in the region of sizeable transmission for single holes. The more recently discovered extraordinary transmission phenomenon was, however, observed for narrower holes (relative to the wavelength), the transmission of which exceeded orders of magnitude the one expected from the sum of their individual transmissions (Ebbesen et al., 1998). For square arrays under normal incidence, a transmission minimum occurred at a wavelength close to the period $a$, coinciding with the Rayleigh condition (Lord Rayleigh, 1907), and a transmission maximum showed up at longer wavelength, thus revealing its connection to Wood's anomalies (Ghaemi et al., 1998; Sarrazin et al., 2003). However, the explanation of the effect is still a subject of debate, as some understand that it originates mainly in the interaction of the apertures with surface plasmons (Ghaemi et al., 1998; Popov et al., 2000; Martín-Moreno et al., 2001; Salomon et al., 2001; Wannemacher, 2001; Barnes et al., 2004), whereas others make emphasis in dynamical light diffraction (Treacy, 1999, 2002; Sarrazin et al., 2003; Lezec and Thio, 2004). While the latter works well to understand the observed extraordinary optical transmission in drilled plasmon-free perfect conductors (Mittra et al., 1988; Gómez-Rivas et al., 2003; Cao and Nahata, 2004; Miyamaru and Hangyo, 2004), supporters of the surfaceplasmon interpretation argue that the enhanced transmission relies in this case on plasmonlike lattice-surfacebound modes sustained by patterned perfect-conductor surfaces (Pendry et al., 2004). Actually, evidence of such modes had been observed before in periodically perforated metallic screens for wavelengths several times larger than the period (Ulrich and Tacke, 1972). We illustrate below how these are in fact complementary views of the same phenomenon and how diffraction in particle arrays contains already the essential features that can be translated to understand the phenomenology of hole arrays. But we first summarize experimental and theoretical findings in this area.

\section{OVERVIEW OF EXISTING RESULTS}

A large amount of literature has been accumulated on transmission through periodic structures, and it is an interesting exercise to reexamine it in connection to recent developments.

\section{A. Single holes}

Bethe's predicted cutoff in the transmission of a single hole in a perfect-conductor thin screen as $(b / \lambda)^{4}$ is the leading-order term in the expansion of the transmission cross section in powers of $b / \lambda$ (Bethe, 1944). Subsequent higher-order analytical corrections (Bouwkamp, 1954; Chang et al., 2006), and eventually rigorous numerical calculations (Roberts, 1987; García de Abajo, 2002), demonstrated that the cross section lies below the hole area up to a radius $b \approx 0.2 \lambda$. These results have found experimental corroboration down to the NIR regime (Obermüller and Karrai, 1995), with new localized plasmon resonances showing up at shorter wavelengths (Degiron et al., 2004; Rindzevicius et al., 2007).

Two different mechanisms have been, however, suggested to achieve enhanced transmission in a single hole: filling it with a material of high permittivity (García de Abajo, 2002; García-Vidal et al., 2005; Webb and Li, 2006), thus creating a partially bound cavity mode that couples resonantly to incident light (see Sec. III.E); and decorating the aperture with periodic corrugations (Lezec et al., 2002) in much the same way as highly directional antennas are capable of focusing electromagnetic radiation on a central dipole element by means of concentric, periodically spaced metallic rings (James, 1977). 


\section{B. Optical transmission through hole arrays}

The intensity of light passing through holes is boosted at certain wavelengths when we arrange them periodically. Pioneering calculations and microwave experiments showed zero reflection in thin films perforated by periodic arrays of small apertures of radius $b \approx 0.36 \lambda$ (Chen, 1971). Further seminal experiments focused on the relation between hole arrays in thin metal screens and their complementary screens (Ulrich, 1967), putting Babinet's principle to a test in the far-infrared region. This was followed by numerous applied studies of hole arrays (regarded as frequency-selective surfaces) in the engineering community, including filters for solar energy collection and elements to enhance antennae performance (Maystre, 1980; McPhedran et al., 1980; Cwik et al., 1987; Mittra et al., 1988).

Ebbesen et al. (1998) demonstrated in the optical domain extraordinary light transmission for openings of radius below the cutoff of the first propagating mode in a circular waveguide, $b<0.29 \lambda$. Since then, this phenomenon has been consistently observed for a varied list of metallic materials (Przybilla, Degiron, et al., 2006), over a wide range of wavelengths [e.g., for microwaves (Gómez-Rivas et al., 2003; Cao and Nahata, 2004), to which metals respond as nearly perfect conductors, in the infrared (Selcuk et al., 2006), and in the vacuum ultraviolet (vuv), using a good conductor in this regime like $\mathrm{Al}$ (Ekinci et al., 2007)], and with different types of array symmetries, including 2D quasicrystal arrangements (Przybilla, Genet, and Ebbesen, 2006; Schwanecke et al., 2006; Sun et al., 2006; Matsui et al., 2007; Papasimakis et al., 2007).

Two examples of enhanced transmission, from Krishnan et al. (2001) and Martín-Moreno et al. (2001), are shown in Fig. 1. The transmission is several times larger in the infrared peak than the prediction of Bethe for noninteracting holes in a thin screen, and four orders of magnitude larger than what is expected for noninteraction apertures in a perfect-conductor film of the same thickness (dashed curves).

Light transmission through hole arrays has been examined theoretically for four decades (Eggimann and Collin, 1962; Chen, 1971; McPhedran et al., 1980, Dawes et al., 1989), although a detailed account of extraordinary optical transmission in real metals had to wait until the new century began (Popov et al., 2000; MartínMoreno et al., 2001; Salomon et al., 2001, Wannemacher, 2001; Sarrazin et al., 2003) and the advance in computation power allowed predictive capacity (Klein Koerkamp et al., 2004; Chang et al., 2005).

The influence of various geometrical and environmental factors has been studied. In particular, the role of hole shape has been shown to yield nontrivial effects (Elliott et al., 2004; Gordon et al., 2004; Klein Koerkamp et al., 2004; Krasavin et al., 2005; van der Molen et al., 2005), such as larger enhancement and redshift of the transmission peaks with respect to the Rayleigh condition for light polarized along the short axis of elongated apertures. Finite arrays exhibit interesting shifts in the

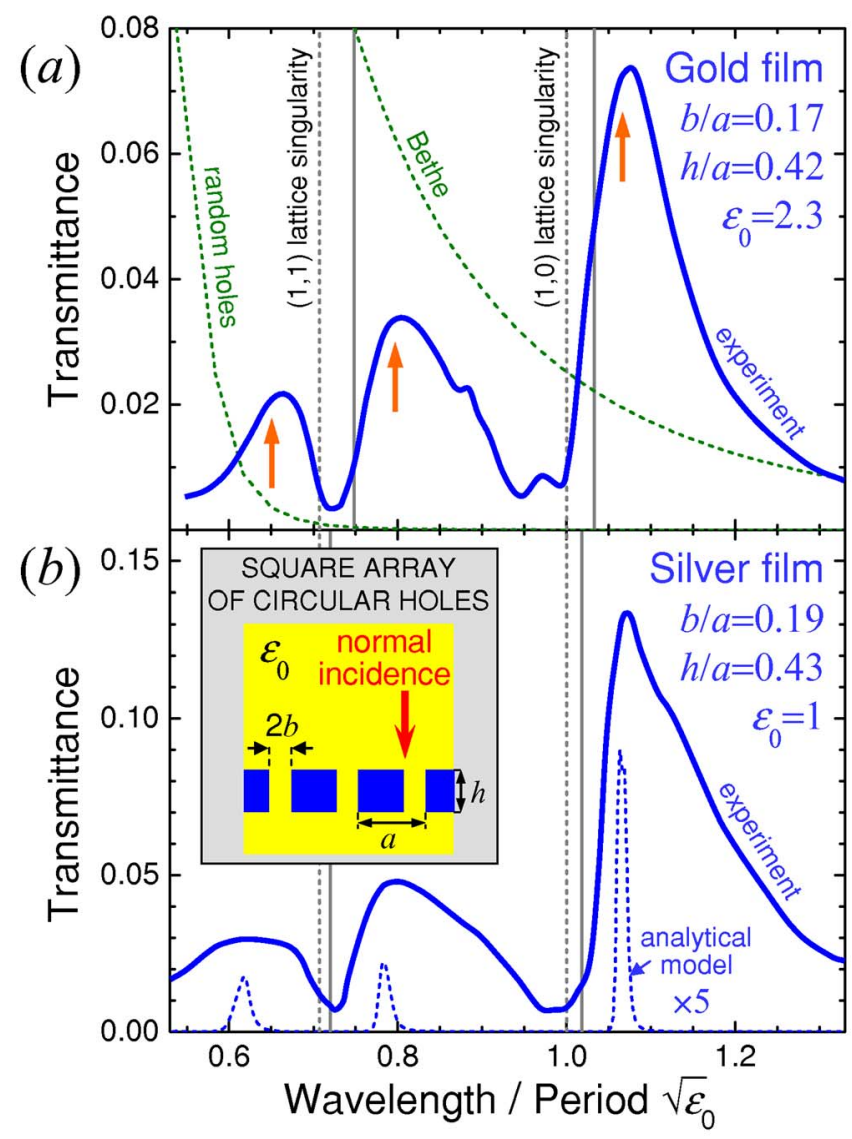

FIG. 1. (Color online) Extraordinary optical transmission in hole arrays. The measured transmittance (solid curves) is shown for apertures drilled in (a) gold and (b) silver films, from Krishnan et al. (2001) and Martín-Moreno et al. (2001), respectively. The silver film is self-standing in air, while the gold film is deposited in quartz and immersed in an index-matching liquid. The lattice constant is $a=600 \mathrm{~nm}$ in Au and $a=750 \mathrm{~nm}$ in Ag. The transmittance of the perforated gold goes well above that predicted for noninteracting apertures in a perfectconductor film or by Bethe's formula for a thin screen (dashed curves). Rayleigh's condition for the $(1,0)$ and $(1,1)$ beams becoming grazing are indicated by vertical dashed lines. Analytical results are shown as arrows in (a) and as a dashed curve in (b) (see Sec. IV.D). The transmittance is presented vs wavelength in the dielectric environment of the metal, normalized to the lattice constant.

transmission maxima as well, depending on the number of apertures (Bravo-Abad et al., 2004; Lezec and Thio, 2004). More exotic shapes like annular holes have also been simulated (Roberts and McPhedran, 1988; Baida and Van Labeke, 2002) and measured (Fan et al., 2005), with the additional appeal that annular waveguides support always one guided mode at least (Jackson, 1999).

The transmission is exponentially attenuated with hole depth because it is mediated by evanescent modes of the apertures regarded as narrow subwavelength waveguides. However, strong signatures of interaction between both metal interfaces have been reported (Degiron et al., 2002), as well as high sensitivity to dielectric environment, so that maximum transmission is achieved 
when the permittivity is the same on the two sides of the film (Krishnan et al., 2001).

Extraordinary optical transmission has expanded to a wide range of phenomena (Genet and Ebbesen, 2007), like the interaction of hole arrays with molecules for potential applications in biosensing (Dintinger, Klein, and Ebbesen, 2006) and all-optical switching (Smolyaninov et al., 2002; Janke et al., 2005; Dintinger, Robel, et al., 2006), and the demonstration of the quantum nature of plasmons through photon entanglement preservation after traversing a hole array (Altewischer et al., 2002).

\section{Particles}

The field of light scattering by small particles has a rich research tradition (van de Hulst, 1981; Bohren and Huffman, 1983) that is being continued by hot topics such as, for example, novel near-field effects in the coupling of metallic nanoparticle arrays (Krenn et al., 1999) and strong interparticle interactions in dimers (Atay et al., 2004; Nordlander et al., 2004; Romero et al., 2006). Here we single out two recent developments in line with the rest of our discussion. The first one refers to coupled metallic nanoparticle arrays. These particles can sustain localized plasmon excitations that hop across neighbors. It has been suggested (Quinten et al., 1998), and later confirmed by experiment (Maier et al., 2001, 2003), that this phenomenon can be utilized to transmit light energy along chains of subwavelength particles, thus providing some basic constituents for future plasmonic devices.

In a different development, the scattering spectra from 1D and 2D arrays of metallic nanoparticles were predicted to exhibit very narrow plasmon line shapes produced by dynamical scattering (Zou and Schatz, 2004; Zou et al., 2004). Experiments performed on lithographically patterned particle arrays confirmed this effect and achieved reasonable control over spectral line shapes (Hicks et al., 2005). We discuss this further in Sec. III.A.2.

\section{TUTORIAL APPROACH}

A tutorial model will be presented next that becomes exact in the limit of narrow holes or small particles in perfect-conductor films. This model describes the basic physics involved both in extraordinary light transmission and in lattice surface modes of structured metals, but leads to simple analytical expressions that permit understanding these phenomena in a fundamental way and making several challenging predictions.

\section{A. Basic relations}

We start with some basic analytical relations for the scattering of an external light plane wave on a periodic array of identical particles that are small compared to both the wavelength and their separation (see Fig. 2). Within linear, nonmagnetic response, the particle at position $\mathbf{R}_{n}$ can be assumed to respond with an induced dipole $\mathbf{p}_{n}=\alpha_{E} \mathbf{E}\left(\mathbf{R}_{n}\right)$, determined by its electric polariz-

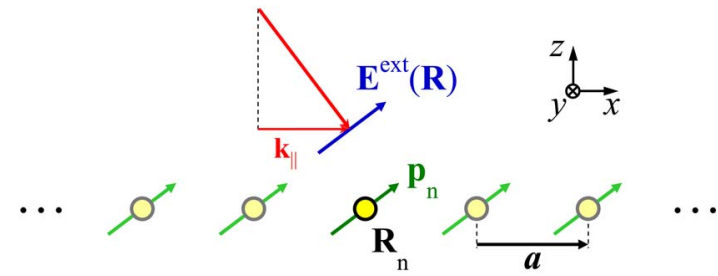

FIG. 2. (Color online) Two-dimensional array of small identical particles illuminated by a light plane wave. $\mathbf{k}_{\|}$is the momentum component parallel to the array. The particle at position $\mathbf{R}_{n}$ displays a dipole $\mathbf{p}_{n}$.

ability tensor $\alpha_{E}$ and the self-consistent field acting on it $\mathbf{E}\left(\mathbf{R}_{n}\right)$. This dipole induces an electric field at point $\mathbf{r}$ that can be written $\mathcal{G}^{0}\left(\mathbf{r}-\mathbf{R}_{n}\right) \mathbf{p}_{n}$ in terms of the dipoledipole interaction tensor,

$$
\mathcal{G}^{0}(\mathbf{r})=\left(k^{2}+\nabla \nabla\right) \frac{e^{i k r}}{r}
$$

where $k$ is the light momentum in free space. ${ }^{1}$ Now, the self-consistent dipole of our particle is found to be

$$
\mathbf{p}_{n}=\alpha_{E}\left[\mathbf{E}^{\mathrm{ext}}\left(\mathbf{R}_{n}\right)+\sum_{n^{\prime} \neq n} \mathcal{G}^{0}\left(\mathbf{R}_{n}-\mathbf{R}_{n^{\prime}}\right) \mathbf{p}_{n^{\prime}}\right],
$$

where $\mathbf{E}^{\operatorname{ext}}\left(\mathbf{R}_{n}\right)=\mathbf{E}^{\operatorname{ext}} \exp \left(i \mathbf{k}_{\|} \cdot \mathbf{R}_{n}\right)$ is the external electric field, which depends upon the site position $\mathbf{R}_{n}$ through a phase factor involving components of the incoming wave momentum parallel to the array $\mathbf{k}_{\|}$, as illustrated in Fig. 2 , and the second term inside the square brackets represents the field induced by the rest of the particles. Bloch's theorem guarantees that the solution of Eq. (2) must have the form $\mathbf{p}_{n}=\mathbf{p} \exp \left(i \mathbf{k}_{\|} \cdot \mathbf{R}_{n}\right)$. Direct insertion of this expression into Eq. (2) leads to

$$
\mathbf{p}=\frac{1}{1 / \alpha_{E}-G\left(\mathbf{k}_{\|}\right)} \mathbf{E}^{\text {ext }}
$$

and

$$
G\left(\mathbf{k}_{\|}\right)=\sum_{n \neq 0} \mathcal{G}^{0}\left(\mathbf{R}_{n}\right) e^{-i \mathbf{k}_{\|} \cdot \mathbf{R}_{n}}
$$

where we have chosen $\mathbf{R}_{0}=0$. Notice that the denominator of Eq. (3) separates the properties of the particles $\left(\alpha_{E}\right)$ from those of the lattice [the structure-factor-type of sum $G\left(\mathbf{k}_{\|}\right)$], in the spirit of the Korringa-KohnRostoka (KKR) method in solid-state physics (Ashcroft and Mermin, 1976). The lattice sum in Eq. (4) can be converted into rapidly converging sums using Ewald's method (Glasser and Zucker, 1980), and we have used in particular the procedure by Kambe (1968).

Incidentally, Eqs. (2)-(4) can be also applied to 3D particle arrays with $\mathbf{k}_{\|}$replaced by a 3D crystal momentum. This type of approach has been shown to lead to robust band gaps in atomic lattices (van Coevorden et al., 1996). Furthermore, Eq. (2) together with the

\footnotetext{
${ }^{1}$ More explicitly, $\quad \mathcal{G}^{0}(\mathbf{r}) \mathbf{p}=\left[\exp (i k r) / r^{3}\right]\left\{\left[(k r)^{2}+i k r-1\right] \mathbf{p}\right.$ $\left.-\left[(k r)^{2}+3 i k r-3\right](\mathbf{r} \cdot \mathbf{p}) \mathbf{r} / r^{2}\right\}$.
} 
Clausius-Mossotti formula (Ashcroft and Mermin, 1976) constitute the basis of the discrete-dipole approximation method for solving Maxwell's equations in arbitrary geometries (Purcell and Pennypacker, 1973; Draine and Flatau, 1994). It should also be noted that the present approach can be extended to larger particles arranged in ordered (Stefanou et al., 1998, 2000) or disordered arrays (García de Abajo, 1999) by including higher-order multipoles, and that this is one of the methods that can be actually applied to deduce effective optical properties of composite materials (Milton, 2002; Romero et al., 2006).

It is useful to represent the dipole-dipole interaction in $2 \mathrm{D}$ momentum space in the plane of the array, which we take to coincide with $z=0$. This is done by expressing the scalar interaction at the right end of Eq. (1) as

$$
\frac{e^{i k r}}{r}=\frac{i}{2 \pi} \int \frac{d^{2} \mathbf{Q}}{k_{z}} e^{i\left(\mathbf{Q} \cdot \mathbf{R}+k_{z}|z|\right)},
$$

where $k_{z}=\sqrt{k^{2}-Q^{2}}$ is the normal momentum and the notation $\mathbf{r}=(\mathbf{R}, z)$, with $\mathbf{R}=(x, y)$, has been adopted. From here and Eq. (1) one obtains expressions like

$$
\mathcal{G}_{x x}^{0}(\mathbf{r})=\frac{i}{2 \pi} \int \frac{d^{2} \mathbf{Q}}{k_{z}}\left(k^{2}-Q_{x}^{2}\right) e^{i\left(\mathbf{Q} \cdot \mathbf{R}+k_{z}|z|\right)}
$$

for the components of the interaction tensor, here specified for the $x x$ directions. This allows us to recast Eq. (4) into a sum over $2 \mathrm{D}$ reciprocal-lattice vectors $\mathbf{g}$, using the relation

$$
\sum_{n} \exp \left(i \mathbf{Q} \cdot \mathbf{R}_{n}\right)=\frac{(2 \pi)^{2}}{A} \sum_{\mathbf{g}} \delta(\mathbf{Q}-\mathbf{g}),
$$

where $A$ is the area of the lattice unit cell. For example, the $G_{x x}$ component under normal incidence $\left(k_{\|}=0\right)$ becomes

$$
\begin{aligned}
G_{x x}(0)= & \lim _{z \rightarrow 0}\left(\frac{2 \pi i}{A} \sum_{\mathbf{g}} \frac{1}{k_{z}^{g}}\left(k^{2}-g_{x}^{2}\right) e^{i k_{z}^{g}|z|}\right. \\
& \left.-\frac{i}{2 \pi} \int \frac{d^{2} \mathbf{Q}}{k_{z}}\left(k^{2}-Q_{x}^{2}\right) e^{i k_{z}|z|}\right),
\end{aligned}
$$

where $k_{z}^{g}=\sqrt{k^{2}-g^{2}}$ and the integral represents the subtraction of the $n=0$ term in the sum of Eq. (4). This expression is important to elucidate some properties of the lattice sums, as we show below.

\section{Reflection and absorption in particle arrays}

The scattered field is given by a Rayleigh expansion similar to the one in Eq. (7) (García de Abajo et al., 2006), with each vector $\mathbf{g}$ labeling one reflected and one transmitted beam of parallel momentum $\mathbf{k}_{\|}+\mathbf{g}$ (Lord Rayleigh, 1907). In the far field in particular, the zeroorder $(\mathbf{g}=0)$ reflection and transmission coefficients under normal incidence reduce to ${ }^{2}$

\footnotetext{
${ }^{2}$ We assume that the array possesses specular symmetry with respect to the $X Z$ plane.
}

$$
r=\frac{2 \pi i k / A}{1 / \alpha_{E}-G_{x x}(0)}
$$

and

$$
t=1+r,
$$

where the first term in the right-hand side of Eq. (9) represents the unscattered beam, and the numerator of Eq. (8) is the far-field amplitude produced by a lattice of unit dipoles.

Interestingly, the absorbance of the array is given by $1-|1+r|^{2}-|r|^{2}$ [see Eq. (9)], which when regarded as a function of the complex variable $r$ has a maximum of $50 \%$ coinciding with $r=-1 / 2$ and $t=1 / 2$. This condition is easily attainable near a lattice singularity (see Sec. III.B), using, for instance, weakly dissipative spherical particles. Similar results have been predicted for narrow cylinder arrays (Laroche et al., 2006), in which $100 \%$ absorption is possible in one of the polarization components for the right choice of parameters.

A particularly simple situation is encountered when the wavelength is larger than the lattice spacing, so that all diffracted beams other than the zero-order beam are evanescent $\left(\left|\mathbf{k}_{\|}+\mathbf{g}\right|>k\right)$. Then, upon inspection of Eq. (7), one finds the useful relation

$$
\operatorname{Im}\left\{G_{x x}(0)\right\}=2 \pi k / A-2 k^{3} / 3, \quad k<g_{1},
$$

where $g_{1}$ denotes the period of the reciprocal lattice ( $g_{1}=2 \pi / a$ for square arrays). Moreover, if the particles are nonabsorbing, the optical theorem constrains their polarizability by the condition $\operatorname{Im}\left\{-1 / \alpha_{E}\right\}=2 k^{3} / 3$ (van de Hulst, 1981). Combining these expressions, one obtains

$$
r=-\frac{1}{1+(i A / 2 \pi k) \operatorname{Re}\left\{1 / \alpha_{E}-G_{x x}(0)\right\}}
$$

for the reflection coefficient of nondissipative particles under normal incidence below the diffraction threshold.

The electrostatic approximation provides a reasonable description of the electric polarizability of small particles $\alpha_{E}^{\text {es }}$. However, this needs to be amended in order to comply with the mentioned optical-theorem constrain, for instance, via the prescription $\alpha_{E}=1 /\left(1 / \alpha_{E}^{\mathrm{es}}-2 i k^{3} / 3\right)$ (Weber and Ford, 2004). Analytical expressions for $\alpha_{E}^{\text {es }}$ exist for a variety of particle shapes, including homogeneous spheres $\left[\alpha_{E}^{\mathrm{es}}=b^{3}(\epsilon-1) /(\epsilon+2)\right.$, where $b$ is the radius and $\epsilon$ is the permittivity] and ellipsoids (Jones, 1945).

We illustrate the applicability of Eq. (11) through an example consisting of square lattices of perfectly conducting thin disks. Figure 3 compares the analytical result of Eq. (11) (dashed curves) with the full solution of Maxwell's equations obtained by following a layer-KKR multiple-scattering formalism (Stefanou et al., 1998, 2000) to simulate the array together with a modal expansion solution of the isolated disk similar to the one available for isolated holes (Roberts, 1987; García de Abajo, Gómez-Medina, and Sáenz, 2005). In the analytical solution we have used the polarizability of thin metallic disks as derived from an ellipsoid of vanishing height, 

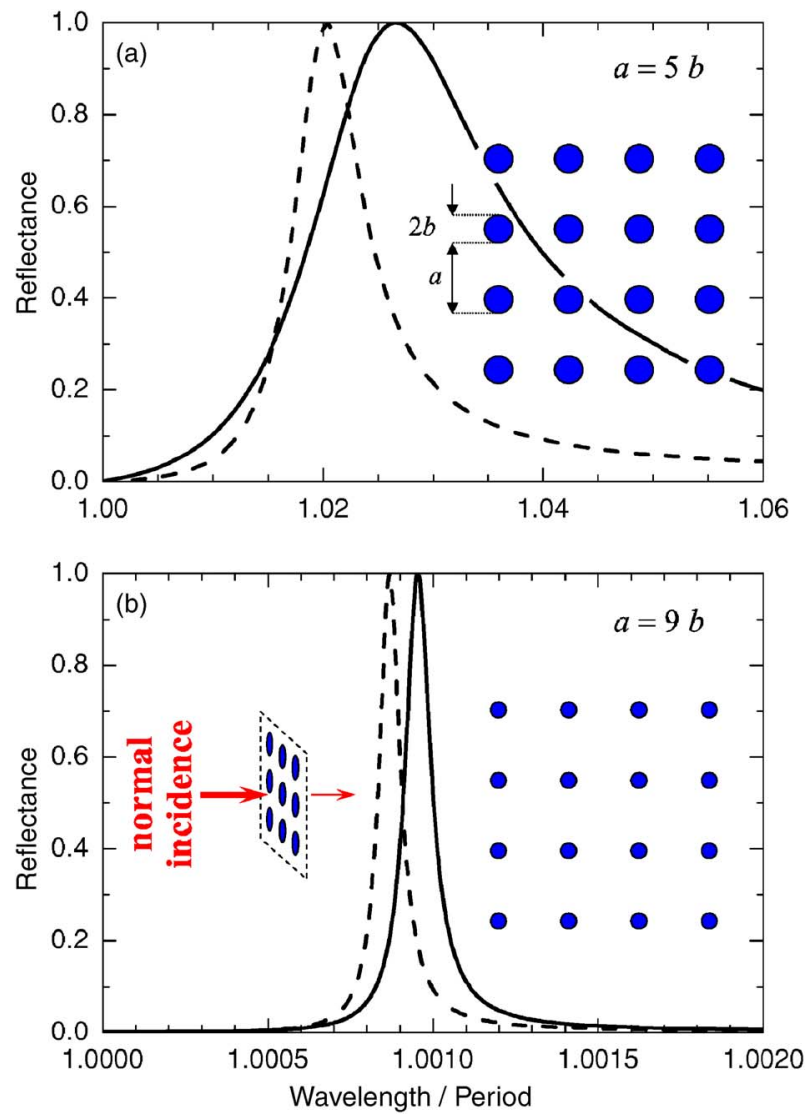

FIG. 3. (Color online) Reflectance spectra of square arrays of perfectly conducting thin circular disks. The wavelength $\lambda$ is normalized to the lattice constant $a$. The disks radius is (a) $b$ $=a / 5$ and (b) $b=a / 9$. Light is impinging normal to the array and $100 \%$ reflection is observed in these two cases at the maximum. Solid curves, full numerical results. Dashed curves, analytical model for $|r|^{2}$ [Eq. (11)].

$\alpha_{E}^{\text {es }}=4 b^{3} / 3 \pi$, where $b$ is the radius. The results of the analytical model describe qualitatively the presence of zero- and full-reflection points in the spectra, irrespectively of the disk size, and we discuss this point further in Sec. III.C.

\section{Narrowing line shapes through dynamical scattering}

The above formalism can be used to explain the effect of narrowed plasmon line shapes in the scattering spectra of 1D and 2D particle arrays (Zou and Schatz, 2004; Zou et al., 2004; Hicks et al., 2005). For simplicity, we discuss metallic spherical particles described by the Drude dielectric function

$$
\epsilon(\omega)=1-\frac{\omega_{p}^{2}}{\omega(\omega+i \eta)},
$$

where $\omega_{p}$ is the bulk plasma frequency and the plasmonamplitude damping rate is $\approx \eta / 2 \ll \omega_{p}$.

Using this expression to obtain the polarizability of a small sphere of radius $b$ (see Sec. III.A.1), we recast Eq. (3) into a Lorentzian of width $\approx \eta / 2+\left(\omega_{p} b^{3} / 2 \sqrt{3}\right) \operatorname{Im}\{G\}$. The natural width of the isolated particles is now supple- mented by a term proportional to $\operatorname{Im}\{G\}$ [see Eq. (10)], which can take negative values that compensate the $\eta / 2$ term to render arbitrarily narrow collective plasmon resonances for an appropriate choice of array parameters.

Applying this to a $2 \mathrm{D}$ square array under normal incidence with $\lambda \sim a$, we find that Eq. (10) yields complete cancellation of the width for $b / a \approx 0.24\left(\eta / \omega_{p}\right)^{1 / 3}$. Under such conditions, the narrowing of the width is limited by the physical requirement that $|r|^{2}+|t|^{2} \leqslant 1$ [see Eqs. (8) and (9)].

\section{B. Lattice singularities}

The interaction among particles in the periodic arrays of Sec. III.A appears to be governed by the lattice sums $G\left(\mathbf{k}_{\|}\right)$and is dominated by their singularities, which originate in accumulation of in-phase scattered fields. Following similar arguments to previous expositions of this idea (Lord Rayleigh, 1907; Fano, 1941), we consider a $1 \mathrm{D}$ periodic chain of particles illuminated by an incident plane wave with both propagation direction and electric field perpendicular to the array, so that the field induced by a given particle on a distant one scales with the inverse of their separation, and thus the contribution of distant particles to the interaction lattice sum has the convergence properties of the series $\sum_{n=1}^{\infty} e^{i k a n} / n$, which diverges as the wavelength approaches the period $a$ as $-\ln |k a-2 \pi|$ (Gradshteyn and Ryzhik, 1980). The same is true for 2D arrays. These singularities in $G\left(\mathbf{k}_{\|}\right)$are signaled by the Rayleigh condition of a diffracted beam becoming grazing (Lord Rayleigh, 1907), as can be seen from Eq. (7), where divergent terms $g \approx k$ (i.e., terms with zero normal momentum $k_{z}^{g}$ ) dominate the sum.

A consequence of this analysis is that the array becomes invisible to the incoming light right at the lattice sum divergence $\left[G_{x x}(0) \rightarrow \infty\right.$, so $r \rightarrow 0$, according to Eq. (8)], showing $100 \%$ transmission even for absorbing particles.

Focusing for simplicity on a square array of period $a$, the normal-incidence lattice sum (7) diverges as (García de Abajo, Gómez-Medina, and Sáenz, 2005)

$$
G_{x x}(0) a^{3} \approx 4 \pi^{2} \sqrt{2} \frac{1}{\sqrt{\lambda / a-1}}-118
$$

for $\lambda \gtrsim a$, where a fitted constant has been subtracted in order to extend the validity of this expression well beyond the singularity.

For oblique incidence with $\mathbf{k}_{\|}$along one of the lattice unit vectors $\| \hat{\mathbf{x}}$, proceeding as in the derivation of Eq. (7), one finds that $G\left(\mathbf{k}_{\|}\right)$is diagonal and its components diverge as

$$
G\left(\mathbf{k}_{\|}\right) \propto \frac{1}{\sqrt{\left(k_{\|}+2 \pi n / a\right)^{2}+(2 \pi l / a)^{2}-k^{2}}},
$$




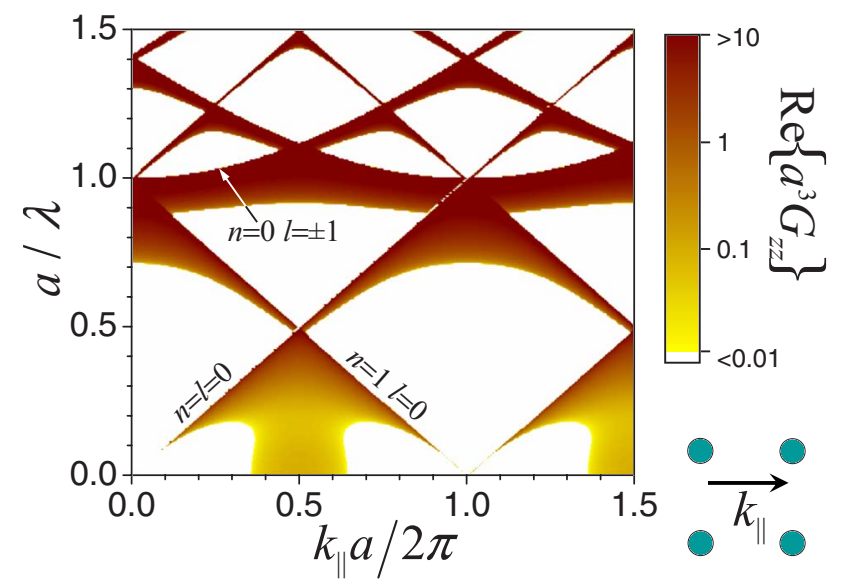

FIG. 4. (Color online) Lattice sum $G_{z z}\left(\mathbf{k}_{\|}\right)[$Eq. (4)] for a square lattice of period $a$ as a function of parallel momentum $k_{\|}$and wavelength $\lambda$. The direction of $\mathbf{k}_{\|}$is along one of the axes of the lattice.

where $n$ and $l$ run over integral numbers (excluding $l$ $=0$ in $\left.G_{x x}\right)$. This behavior is illustrated in Fig. 4, showing the lattice singularities exhibited by $\operatorname{Re}\left\{G_{z z}\left(\mathbf{k}_{\|}\right)\right\}$.

\section{Hole arrays}

\section{Babinet's principle and hole arrays in thin screens}

The behavior of hole arrays in perfect-conductor screens can be directly connected to the properties of the disk arrays considered in Fig. 3. Indeed, one can invoke the exact Babinet principle (Born and Wolf, 1999; Jackson, 1999), which connects the reflected fields of the disk array for a given incident polarization with the transmitted fields of its complementary hole array with orthogonal polarization, as illustrated in Fig. 5 (García de Abajo, Gómez-Medina, and Sáenz, 2005). Therefore the reflectance spectra shown in Fig. 3 are identical with the transmittance spectra of the complementary perforated screens.

Focusing again on square arrays and normal incidence, we observe two characteristic features in the transmittance spectra: (i) the transmission vanishes when the wavelength $\lambda$ equals the period $a$, and (ii) a $100 \%$ transmission maximum takes place at a wavelength

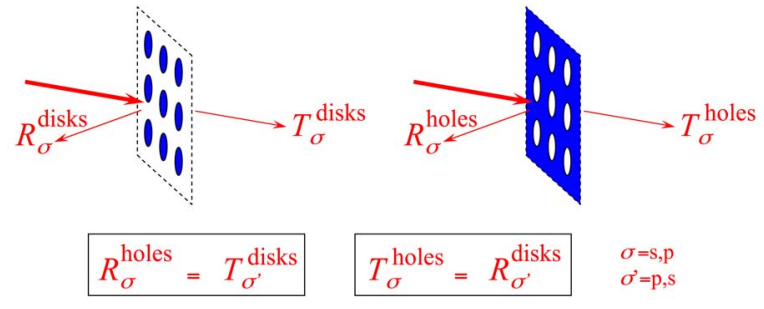

FIG. 5. (Color online) Babinet's principle applied to disk and hole arrays. The transmittance (reflectance) of the disk array for light of a given polarization $\sigma$ ( $s$ or $p$ ) is identical to the reflectance (transmittance) of the complementary hole array for orthogonal polarization $\sigma^{\prime}$ ( $p$ or $s$, respectively).

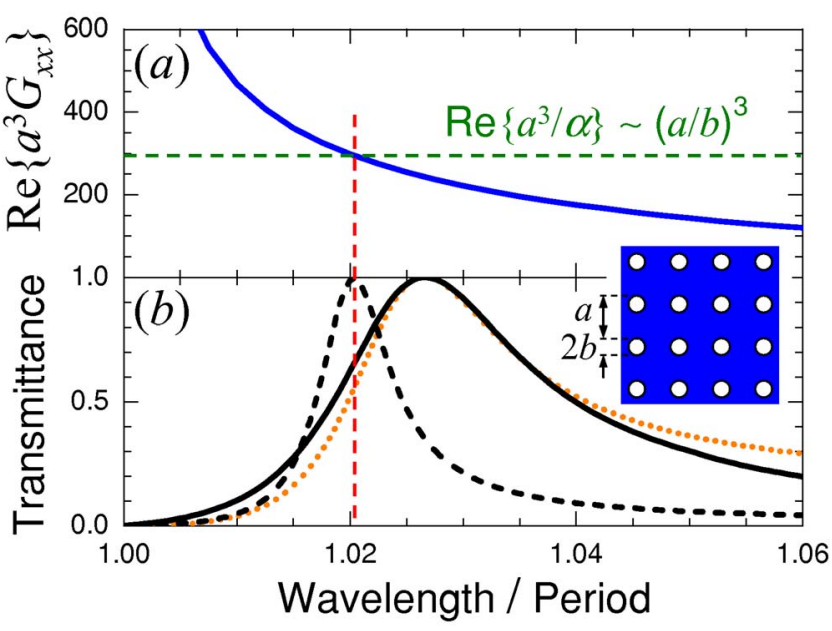

FIG. 6. (Color online) Geometrical construction of the condition of full transmission in a hole array. (a) Wavelength dependence of the real part of the lattice sum $G_{x x}$ [Eq. (4)] for $k_{\|}$ $=0$. (b) Normal-incidence transmittance of a hole array complementary of the disk array of Fig. 3(a) $(b=a / 5)$ : exact calculation (solid curve), analytical model of Eq. (11) (dashed curve), and Fano profile of Eq. (15) (dotted curve). The transmission minimum at $\lambda=a$ results from the divergence of $G_{x x}$, while the transmission maximum (see vertical dashed line) is derived from the condition that $\operatorname{Re}\left\{G_{x x}\right\}$ equals the inverse of the hole polarizability, according to Eq. (11).

slightly above $a$. The origin of these effects can be traced back to Wood's anomalies in gratings (Wood, 1902, 1912, $1935)$ and to their interpretation in terms of the following two mechanisms (Fano, 1936, 1941): (i) accumulation of in-phase scattering events when the wavelength equals the period (see explanation in Sec. III.B), and (ii) coupling of the incident light to a surface resonance. These phenomena persist in hole arrays perforated in thicker films of nonideal absorbing metals, for which the maximum transmission is reduced but justifies the term extraordinary optical transmission (Ebbesen et al., 1998).

The analytical simplicity of the transmission coefficient for our thin-screen hole array, given by the righthand side of Eq. (11), allows deeper insight into the origin of this phenomenon. The lattice sum $G_{x x}(0)$ was shown to diverge when $\lambda=a$, as Fig. 6 illustrates. This leads to vanishing transmission, which we can interpret in terms of accumulation of in-phase scattering (see discussion in Sec. III.B). Furthermore, 100\% transmission is achieved if the second term in the denominator of Eq. (11) becomes zero, a condition that can be rigorously fulfilled for arbitrarily tiny apertures (García de Abajo, Gómez-Medina, and Sáenz, 2005): the smaller the holes, the larger $1 / \alpha_{E}$, because the polarizability is proportional to the cube of their radius, but no matter how large this fraction becomes, there is always one wavelength at which the divergent lattice sum matches it. This statement is illustrated by geometrical construction in Fig. 6, in which the point of intersection of the horizontal dotted line and the solid curve [Fig. 6(a)] signals 
the condition $\operatorname{Re}\left\{1 / \alpha_{E}-G_{x x}(0)\right\}=0 .^{3}$ The possibility of $100 \%$ transmission in nonabsorbing structures has already been pointed (Maystre, 1980; McPhedran et al., 1980), and the theory presented goes further to show that this is possible for arbitrarily small holes. Nevertheless, the number of apertures needed to accomplish high transmission will increase as they become smaller, and at the same time the transmission resonance will be increasingly narrower and closer to $\lambda=a$. Therefore these transmission maxima involve long-range interaction among holes, dominated by dynamical diffraction (i.e., multiple-scattering paths). In fact, if only single scattering were considered, Eq. (3) would become $\mathbf{p}=\alpha_{E}[1$ $\left.+\alpha_{E} G\left(\mathbf{k}_{\|}\right) \alpha_{E}\right] \mathbf{E}^{\text {ext }}$, which wrongly predicts simultaneous divergence of transmittance and reflectance at $\lambda=a$.

This collective response in planar periodic arrays can be regarded as a lattice surface resonance (Fano, 1941), which becomes a true surface-bound state when evanescent incoming waves are considered, as we explain in Sec. III.D. However, the resonance is strongly coupled to propagating light for external plane-wave illumination, a situation described by Fano (1961) studing a discrete resonance state (our lattice surface-bound mode) coupled to a continuum (the transmitted light). This type of approach has been shown to work rather well in theory (Sarrazin et al., 2003; Chang et al., 2005) and in comparison with measured transmission spectra (Genet et al., 2003). Our transmittance calculations should also respond to Fano profiles of the form (Fano, 1961)

$$
T=C \frac{(q+\varepsilon)^{2}}{1+\varepsilon^{2}}
$$

where $\varepsilon$ can be assimilated to the light frequency and $q$ is the strength of the coupling to the lattice surface resonance. Figure 6(b) compares our exact calculation of the transmittance (solid curve) with a Fano profile corresponding to parameters $q=-3$ and $C=0.1$ (dotted curve), in which we assume a linear relationship between $\varepsilon$ and the light frequency, with $\varepsilon=-0.33 \quad(\varepsilon=3)$ for $T=1 \quad(T$ $=0$ ). The agreement is reasonable, considering that no dependence of the coupling parameter on wavelength is taken into account. This further supports an interpretation of extraordinary transmission in terms of coupling to the lattice surface resonance set up by dynamical diffraction in the array.

The geometrical construction of Fig. 6 provides a visual explanation of transmission in arrays of elongated apertures: an elongated piece of planar metal (e.g., a rectangle) has larger electric polarizability along its longaxis direction, and this has direct consequences for the Babinet-related situation of an elongated hole with the electric field along the short axis; larger polarizability

\footnotetext{
${ }^{3}$ We rely here on the condition $\operatorname{Re}\left\{1 / \alpha_{E}\right\}>0$, which is satisfied by the polarizability of planar, perfectly conducting disks. Interestingly, lattice resonances will be absent in arrays of particles with negative polarizability, such as metallic nanoparticles under blue-detuned illumination relative to a nearby plasmon band.
}

involves more redshifted and broader transmission maxima [this is because the point of crossing in Fig. 6(a) occurs where $G_{x x}$ is less steep], as observed experimentally (Gordon et al., 2004; Klein Koerkamp et al., 2004).

Incidentally, Eqs. (3) and (4) constitute a good approximation to describe the extraordinary transmission observed in 2D quasicrystal hole arrays (Przybilla, Genet, and Ebbesen, 2006; Schwanecke et al., 2006; Sun et al., 2006; Matsui et al., 2007; Papasimakis et al., 2007), in which the lattice sum $G$ exhibits pronounced, but finite maxima related to bright spots in the Fourier transform of the hole distribution. These spots define the reciprocal lattice for periodic arrays, but have quasicrystal angular symmetry in quasicrystals. In the spirit of Rayleigh's explanation of Wood's anomalies (Lord Rayleigh, 1907), the cumulative effect of long-distance interaction among apertures can be claimed to create these reciprocal-space hot spots, so that the effect of neighboring holes can be overlooked and an effective homogeneous $\mathbf{p}$ describes qualitatively the extraordinary transmission effect in quasicrystal arrays (Papasimakis et al., 2007), as well as the rich Talbot-like structure and subwavelength light localization observed at distances up to several wavelengths away from the array (Huang et al., 2007).

\section{Single holes in thick films}

Our use of Babinet's principle indicates that, similar to small particles, small holes in perfect conductors can be assimilated to equivalent induced dipoles, in line with Bethe's pioneering description of the field scattered by a single aperture in a thin screen (Bethe, 1944), which he regarded as arising from a magnetic dipole parallel to the screen plus an electric dipole perpendicular to it.

Narrow holes can still be represented by induced dipoles in thick screens, as illustrated in Fig. 7(a). Parallel electric dipoles and perpendicular magnetic dipoles are forbidden by the condition that the parallel electric field and the perpendicular magnetic field vanish at a perfectconductor surface. This allows defining electric $(E)$ and magnetic $(M)$ polarizabilities both on the same side as the applied field $\left(\alpha_{\nu}\right.$, with $\left.\nu=E, M\right)$ and on the opposite side $\left(\alpha_{\nu}^{\prime}\right)$. Furthermore, energy flux conservation under arbitrary illumination leads to an exact optical-theorem type of relationship between these polarizabilities (García de Abajo et al., 2006): by considering two plane waves incident on either side of the film and by imposing that the incoming energy flux equals the outgoing one (because perfect conductors cannot absorb energy), we obtain the condition

$$
\operatorname{Im}\left\{g_{\nu}^{ \pm}\right\}=\frac{-2 k^{3}}{3}
$$

where 

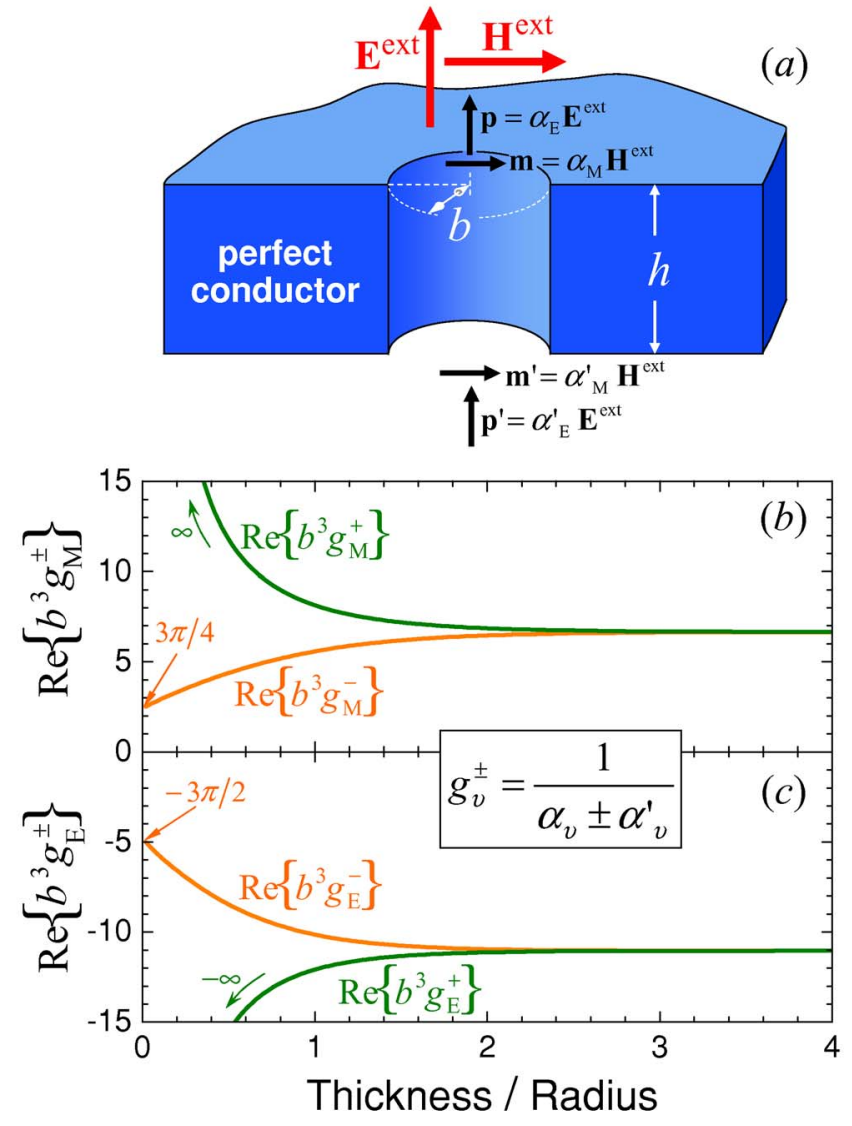

FIG. 7. (Color online) Response of a small hole in a perfectconductor thick film. (a) The field scattered by a subwavelength aperture in response to external electric $\left(E^{\mathrm{ext}}\right)$ and magnetic $\left(H^{\text {ext }}\right)$ fields is equivalent (at large distance compared to the radius $b)$ to that of effective electric $(p)$ and magnetic $(m)$ dipoles, which allow defining polarizabilities $\left(\alpha_{E}\right.$ and $\alpha_{M}$, respectively) both on the same side as the external fields $\left(\alpha_{\nu}\right)$ and on the opposite side $\left(\alpha_{\nu}^{\prime}\right)$. Only the perpendicular component of the electric field and the parallel component of the magnetic field can be nonzero at the surfaces of the perfect-conductor film. (b),(c) Thickness dependence of the real part of the hole response functions $g_{\nu}^{ \pm}$for $\lambda \gg b$ [the imaginary part satisfies Eq. (16)].

$$
g_{\nu}^{ \pm}=\frac{1}{\alpha_{\nu} \pm \alpha_{\nu}^{\prime}}
$$

is defined as hole response functions. The remaining real parts of $g_{v}^{ \pm}$are obtained numerically from the field scattered by a single hole (Roberts, 1987; García de Abajo, 2002). These functions are represented in Figs. 7(b) and $7(\mathrm{c})$ within the electrostatic limit, showing $\left|\operatorname{Re}\left\{g_{\nu}^{+}\right\}\right| \rightarrow \infty$ in the thin-film limit, where $\alpha_{\nu}^{\prime}=-\alpha_{\nu}$ (Jackson, 1999).

\section{Hole arrays in thick films}

Periodic arrays of sufficiently narrow and spaced holes can also be described by perpendicular electric dipoles $p$ and $p^{\prime}$ and parallel magnetic dipoles $m$ and $m^{\prime}$, where primed (unprimed) quantities are defined on the entry (exit) side of the film, as determined by the incoming light [see Fig. 7(a)]. We consider first a unit-electric-field $p$-polarized plane-wave incident on a hole array with parallel momentum $\mathbf{k}_{\|}$along $\hat{\mathbf{x}}$, so that the external field (incident plus reflected) in the absence of the apertures has parallel magnetic field $H_{y}^{\text {ext }}=2$ along the $y$ direction and perpendicular electric field $E_{z}^{\text {ext }}=-2 k_{\|} / k$ along $z$. Then, one can generalize Eq. (3) and write a set of multiple-scattering equations for the self-consistent dipoles (Collin and Eggimann, 1961; Eggimann and Collin, 1962). Symmetry considerations demand that our magnetic and electric dipoles be oriented as $\mathbf{m}=m \hat{\mathbf{y}}$ and $\mathbf{p}$ $=p \hat{\mathbf{z}}$, respectively. Following the notation of Sec. III.A, we can write

$$
\begin{aligned}
& p=\alpha_{E}\left(E_{z}^{\mathrm{ext}}+G_{z z} p-H m\right)+\alpha_{E}^{\prime}\left(G_{z z} p^{\prime}-H m^{\prime}\right), \\
& p^{\prime}=\alpha_{E}^{\prime}\left(E_{z}^{\mathrm{ext}}+G_{z z} p-H m\right)+\alpha_{E}\left(G_{z z} p^{\prime}-H m^{\prime}\right), \\
& m=\alpha_{M}\left(H_{y}^{\mathrm{ext}}+G_{y y} m-H p\right)+\alpha_{M}^{\prime}\left(G_{y y} m^{\prime}-H p^{\prime}\right), \\
& m^{\prime}=\alpha_{M}^{\prime}\left(H_{y}^{\mathrm{ext}}+G_{y y} m-H p\right)+\alpha_{M}\left(G_{y y} m^{\prime}-H p^{\prime}\right),
\end{aligned}
$$

with a new lattice sum defined as

$$
H=-i k \sum_{n \neq 0} e^{-i k_{\|} x_{n}} \partial_{x_{n}} \frac{e^{i k R_{n}}}{R_{n}}
$$

This sum stands for the interaction between mixed electric and magnetic dipoles. We can understand the above equations in an intuitive way; for instance, the first one of them states that the electric dipole on the entry side (p) results from the response to the $z$ component of the self-consistent field on that side $\left(E_{z}^{\mathrm{ext}}+G_{z z} p-H m\right)$ via the polarizability $\alpha_{E}$ plus the response to the selfconsistent field on the opposite film side $\left(G_{z z} p^{\prime}-H m^{\prime}\right)$ via $\alpha_{E}^{\prime}$. The solution to these equations can be written as

$$
\begin{aligned}
& p \pm p^{\prime}=-2\left[\left(g_{M}^{ \pm}-G_{y y}\right) k_{\|} / k+H\right] / \Delta_{ \pm}, \\
& m \pm m^{\prime}=2\left[\left(g_{E}^{ \pm}-G_{z z}\right)+H k_{\|} / k\right] / \Delta_{ \pm}
\end{aligned}
$$

with

$$
\Delta_{ \pm}=\left(g_{E}^{ \pm}-G_{z z}\right)\left(g_{M}^{ \pm}-G_{y y}\right)-H^{2}
$$

The zero-order transmittance of the film with holes is then obtained from the far field set up by the infinite 2D array of induced dipoles, $T_{p}=\mid\left(2 \pi k^{2} / A k_{z}\right)\left(m^{\prime}\right.$ $\left.-p^{\prime} k_{\|} / k\right)\left.\right|^{2}$, where $k_{z}=\sqrt{k^{2}-k_{\|}^{2}}$.

Similar considerations for $s$-polarized light show that its transmittance reduces to $T=\left|2 \pi \mathrm{km}^{\prime} / A\right|^{2}$, with magnetic dipoles parallel to $\mathbf{k}_{\|}$and no electric dipoles whatsoever $\left(E_{z}^{\text {ext }}=0\right)$. More precisely, $m \pm m^{\prime}=\left(2 k_{z} / k\right) /\left(g_{M}^{ \pm}\right.$ $-G_{x x}$ ), from which one obtains 


$$
\begin{aligned}
T_{s}= & \left(\frac{2 \pi k_{z}}{A}\right)^{2}\left|\frac{1}{g_{M}^{+}-G_{x x}}-\frac{1}{g_{M}^{-}-G_{x x}}\right|^{2} \\
= & \mid \frac{1}{1+i A / 2 \pi k_{z} \operatorname{Re}\left\{g_{M}^{+}-G_{x x}\right\}} \\
& -\left.\frac{1}{1+i A / 2 \pi k_{z} \operatorname{Re}\left\{g_{M}^{-}-G_{x x}\right\}}\right|^{2}
\end{aligned}
$$

for the transmittance. The last identity in Eq. (19) comes from Eqs. (10) and (16) for diffractionless arrays.

Interestingly, Eq. (19) predicts $100 \%$ transmission if

$$
1+\left(\frac{A}{2 \pi k_{z}}\right)^{2} \operatorname{Re}\left\{g_{M}^{+}-G_{x x}\right\} \operatorname{Re}\left\{g_{M}^{-}-G_{x x}\right\}=0 .
$$

This is a second-order algebraic equation in $\operatorname{Re}\left\{G_{x x}\right\}$ that admits positive real solutions provided

$$
\frac{A}{4 \pi k_{z}}\left|g_{M}^{+}-g_{M}^{-}\right| \geqslant 1
$$

Actually, $\operatorname{Re}\left\{G_{x x}\right\}$ can match those roots near the $l \neq 0$ singularities of Eq. (14), where it can be chosen arbitrarily large within a narrow range of wavelengths [see Eq. (13)]. It should be noted that the difference $g_{M}^{+}$ $-g_{M}^{-}$falls off rapidly to zero when the film thickness $h$ is made much larger than the hole radius $b$ [see Fig. 7(b)]. However, if we fix both the $h / b$ ratio and the angle of incidence, the left-hand side of Eq. (21) reduces to a positive real constant times $\lambda A / b^{3}$, leading to the conclusion that $100 \%$ transmission is attainable at a wavelength close to the Rayleigh condition (e.g., $\lambda \gtrsim a$ for normal incidence on a square lattice of spacing $a$ ) regardless how narrow the holes are as compared to the film thickness. Surprisingly, this requires that the ratio of the lattice constant to the hole radius be increased for deeper holes in order to compensate the fall in $g_{M}^{+}-g_{M}^{-}$ for larger $h / b$.

The transmittance shows a complex dependence on film thickness $h$ (Martín-Moreno et al., 2001), as illustrated in Fig. 8. The maximum of Fig. 6 is initially blueshifted closer to $\lambda=a$ for small $h$, accompanied by a second narrower peak at even shorter wavelengths ${ }^{4}$ [these are the two solutions of Eq. (20) under the condition (21)]. As $h$ increases, interside interaction weakens and the two $100 \%$ maxima approach each other. At some point only one transmission maximum is observed when the left-hand side of Eq. (21) is exactly 1. For even thicker films, the condition (21) cannot be met any longer and the transmission maximum departs from $100 \%$. The Fano character of these lattice resonances is

\footnotetext{
${ }^{4}$ In fact, there are two lattice resonances for $h=0$, which in the language of Fano arise from coupling to different light continua on either side of the film, but one of these resonances has vanishing width and is placed at $\lambda=a$ due to strong interside interaction.
}

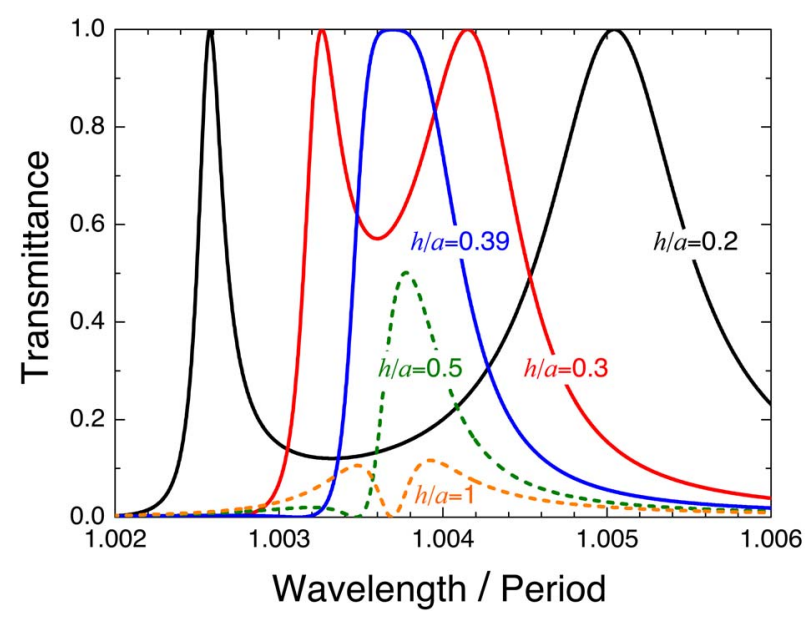

FIG. 8. (Color online) Thickness dependence of the normalincidence transmittance spectra of square arrays of circular holes drilled in perfect-conductor films, according to Eq. (19). The hole radius $b=0.2 a$, the wavelength $\lambda$, and the film thickness $h$ are given relative to the period $a$ (see insets).

again visible through vanishing transmission at a wavelength immediately below the maximum (see $h / a=0.5$ in Fig. 8).

Incidentally, perfect conductors are perfectly nonlossy, so that light dissipation must take place only at the openings if they are infiltrated with some dissipative material. For deep enough holes, the transmission is negligible and the absorbance becomes $1-|r|^{2}$, which can reach $100 \%$ values under suitable resonant conditions, for instance in the IR by combining holes drilled in noble metals (behaving nearly as perfect conductors) infiltrated with phonon-polariton materials. In fact, a similar effect has been observed in the visible using Au gratings (Hutley and Maystre, 1976) and in the infrared using $\mathrm{SiC}$ gratings (Greffet et al., 2002).

\section{Lattice surface modes in structured metals}

The flourishing area of plasmonics is demonstrating how confining electromagnetic fields to a surface can find many potential applications on the nanoscale (Ozbay, 2006). Zenneck waves at radio frequencies (Zenneck, 1907; Barlow, 1958), phonon polaritons in the infrared (Greffet et al., 2002; Hillenbrand et al., 2002), and plasmons in the visible are in fact different manifestations of the same phenomenon: confinement of electromagnetic fields to curved or planar surfaces. Even perfect-conductor screens, which are unable to trap light when they are flat, were experimentally shown by Ulrich and Tacke (1972) to host confined surface modes of $p$ polarization when molded into films pierced by periodic arrays of holes spaced a distance much smaller than the wavelength [see Fig. 9(b)].

In a recent independent development, Pendry et al. (2004) studied surface modes in drilled semi-infinite metal, suggesting the possibility to extend plasmonlike behavior to lower-frequency domains via the flattening of the mode dispersion relation driven by propagating 


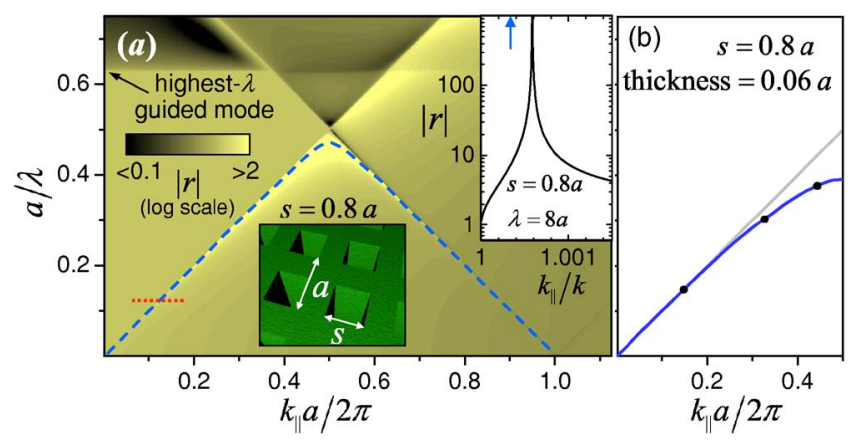

FIG. 9. (Color online) Lattice surface modes in a perforated perfect conductor. (a) The contour plot shows the modulus of the specular reflection coefficient of a semi-infinite metal for incident $p$-polarized light as a function of wavelength $\lambda$ and parallel momentum $k_{\|}$(see insets for parameters). The upperright inset shows a detail of the reflectivity as compared to the mode position predicted by Eqs. (23) and (24) (see arrow). A reflection coefficient larger than 1 is only possible for evanescent waves outside the light cone. (b) Lattice modes in a perforated thin film, as measured by Ulrich and Tacke, 1972 (symbols).

modes of the holes, and stimulating new microwaves observations (Hibbins et al., 2005). The analysis of Pendry et al. (2004) relied on a description of the holes based upon their lowest-order guided modes (i.e., $\mathrm{TE}_{1,0}$ modes), which allowed extracting local permittivity and permeability functions in a metamaterial approach to holey metals. However, García de Abajo and Sáenz (2005) showed later that higher-order modes (and in particular TM modes) are important, giving rise to large quantitative modifications to the dispersion relation and revealing finer details in the metal response with holes that go beyond a simple local metamaterial description (e.g., the angular dependence of the reflection coefficient does not follow the Fresnel equations with local optical constants).

At variance with planar perfect conductors and their lack of surface modes, corrugated metallic surfaces can support bound states even in the long-wavelength limit. In an intuitive picture, surface confinement in a drilled semi-infinite perfect conductor can be related to the evanescent penetration of the electromagnetic field inside the holes, in much the same way as surface plasmons enter a distance of the order of the skin depth inside a metal in the visible and NIR regimes (Barnes and Sambles, 2004). Actually, these modes share with plasmons their character of $p$-polarized evanescent waves.

Next, we elaborate a tutorial, analytical formulation of this phenomenon that becomes exact in the limit of small holes of size $s \ll a \ll \lambda$, arranged in a square lattice of period a (García de Abajo and Sáenz, 2005). Although we focus our analysis on periodic hole arrays drilled in a semi-infinite perfect conductor, it must be emphasized that periodicity is not really needed and that similar modes should exist for patterns other than holes (e.g., small protuberances or particles deposited on an otherwise flat surface).
Using the formalism of Sec. III.C, we find that Eqs. (17) and (18) offer a simple description of lattice surfacebound modes in metallic films. For infinitely deep square holes as sketched in an inset of Fig. 9(a), the surface modes must correspond to nonvanishing values of the induced dipoles $p$ and $m$ in the absence of external fields. This can only be accomplished if the denominator $\Delta_{ \pm}$is zero in those equations, leading to

$$
\left(1 / \alpha_{E}-G_{z z}\right)\left(1 / \alpha_{M}-G_{y y}\right)=H^{2},
$$

where we have set $\alpha_{\nu}^{\prime}=0$ for infinitely deep holes (see Fig. 7). The interaction sums $G_{y y}, G_{z z}$, and $H$ are generally small for $s \ll a$, except near the lattice singularities discussed in Sec. III.B. In particular, near the light line for $k_{\|} \gtrsim k$, one has

$$
\operatorname{Re}\left\{G_{z z}\right\} \approx \operatorname{Re}\left\{G_{y y}\right\} \approx \operatorname{Re}\{H\} \approx \frac{2 \pi k^{2}}{a^{2} \sqrt{k_{\|}^{2}-k^{2}}},
$$

which corresponds to Eq. (14) with $n=l=0$. Furthermore, upon inspection of an expansion for $H$ similar to Eq. (7), we find $\operatorname{Im}\{H\}=0$ outside the light cone, $k_{\|}>k$, and the remaining imaginary parts of all quantities in Eq. (22) cancel out exactly because $\operatorname{Im}\left\{G_{j j}\right\}=\operatorname{Im}\left\{\alpha_{\nu}^{-1}\right\}$ $=-2 k^{3} / 3$ in that region. Combining these results, we obtain an approximate long-wavelength dispersion relation from Eq. (22):

$$
k_{\|}^{2}=k^{2}+\Gamma \frac{S^{3} k^{4}}{a^{4}}
$$

with

$$
\Gamma=\frac{4 \pi^{2}}{S^{3}}\left(\frac{1}{\operatorname{Re}\left\{1 / \alpha_{E}\right\}}+\frac{1}{\operatorname{Re}\left\{1 / \alpha_{M}\right\}}\right)^{2} .
$$

Equation (24) is exact in the $s \ll a \ll \lambda$ limit, and predicts the existence of lattice surface-bound modes under the condition $1 / \operatorname{Re}\left\{1 / \alpha_{E}\right\}+1 / \operatorname{Re}\left\{1 / \alpha_{M}\right\}>0$. Here we have used the area of each hole $S$ to make $\Gamma$ dimensionless.

Calculated values of $\Gamma$ are offered in Fig. 10(c) for various hole geometries. The polarizability $\alpha_{E}\left(\alpha_{M}\right)$ is obtained from the electrostatic (magnetostatic) far field induced by an external electric (magnetic) field, as shown in Fig. 10(a) [Fig. 10(b)]. Interestingly, circular and square openings of the same area give rise to similar values of $\Gamma$. This parameter increases by an order of magnitude when the holes are made on thin screens instead of semi-infinite metals, producing lattice surface modes that are further apart from the light line (see Ulrich and Tacke, 1972), and therefore, more confined to the metal, as a result of cooperative interaction between both sides of the film [see analytical solutions for circular apertures (Jackson, 1999) in last column of Fig. 10(c)]. Another suggestive possibility is offered by split annular holes, which present resonant electric polarizability (Falcone et al., 2004), and by holes filled with high-permittivity materials (see Sec. III.E), for which the interaction with single-hole modes produces large departures of the extended surface states from the grazing light condition. 


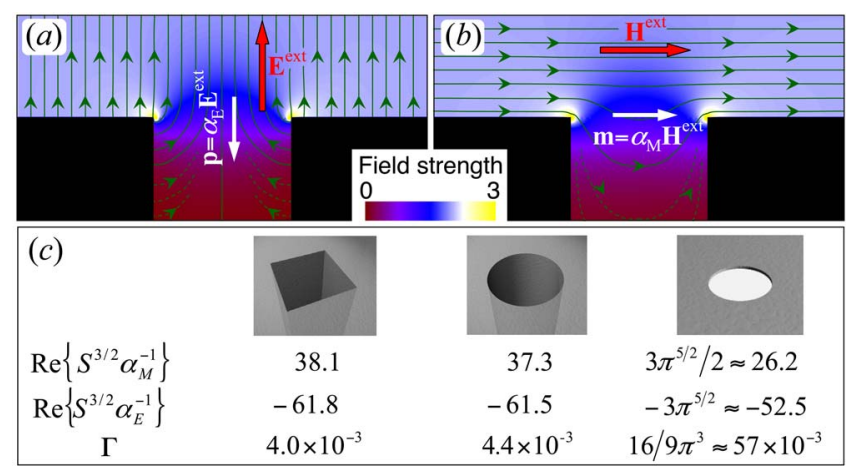

FIG. 10. (Color online) Polarization of holes in perfect conductors. (a) Electrostatic electric-field flow lines for a circular hole drilled in a semi-infinite perfect conductor subject to an external field $\mathbf{E}^{\text {ext }}$ perpendicular to the surface, giving rise to an electric dipole $p=\alpha_{E} E^{\text {ext }}$ as seen from afar. (b) Magnetostatic magnetic-field flow lines for the same hole subject to an external parallel field $\mathbf{H}^{\text {ext }}$ and leading to a magnetic dipole $m=\alpha_{M} H^{\text {ext }}$. (c) Summary of polarizabilities for square and circular holes in perfect-conductor surfaces, normalized using the aperture area $S$. The values for the circular hole are taken from the $h \gg b$ limit of Fig. 7. The circular opening in a thin screen is analytical (Bethe, 1944; Jackson, 1999), but we must correct the right-hand side of Eq. (24) by a factor of 4 in this case because of cooperative interaction between both sides of the film.

Figure 9(a) shows calculated results for the reflection coefficient of a drilled metal, obtained by rigorous solution of Maxwell's equations in which we use a planewave expansion of the field outside the metal and a guided-mode expansion inside the holes (García de Abajo and Sáenz, 2005). The lattice surface mode can be observed as a bright region with a dashed line showing the position at which the reflection coefficient becomes infinite. A detail of $|r|$ for a specific wavelength (see dot-

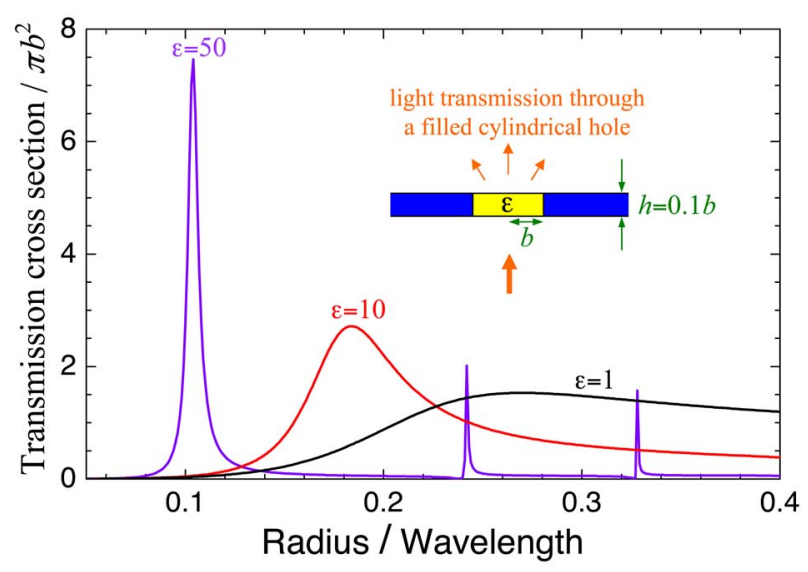

FIG. 11. (Color online) Enhanced transmission driven by a localized resonance. The normal-incidence transmission of a circular aperture drilled in a perfect-conductor film and filled with dielectric material is represented for different values of the permittivity $\epsilon$ (see labels). The transmitted power is normalized to the incoming flux within the hole area. Evidence of Fabry-Perot resonances is clear in the $\epsilon=50$ spectrum. ted straight line) is shown in the inset. The position of the resonance predicted by Eqs. (23) and (24) (see arrow in the inset) is in reasonably close agreement with the exact calculation, considering that the analytical model neglects neighboring-hole multipolar interaction, which is important for openings occupying $64 \%$ of the surface. Finally, Fig. 9(b) shows experimental results for a drilled thin film from Ulrich and Tacke (1972). These surface modes are more bound in perforated thin films than in semi-infinite metals, as can be seen from the values of $\Gamma$ given in Fig. 10(c). Actually, the measured dispersion relation departs substantially from the light line close to the boundary of the first Brillouin zone.

\section{E. Interplay between lattice and site resonances}

The description of extraordinary optical transmission in terms of quasibound surface states driven by lattice singularities can be extended to other types of binding. In particular, a single hole filled with a dielectric of high permittivity can trap light in its interior, giving rise to cavity modes even for subwavelength apertures, provided the permittivity is sufficiently large to shrink the wavelength inside the dielectric to a value comparable to the diameter of the hole. This concept is explored in Fig. 11, in which higher permittivities are seen to produce larger contraction of the wavelength inside the hole, so that the cavity mode condition is met at longer free-space wavelengths for fixed aperture size (García de Abajo, 2002; García-Vidal et al., 2005). This process is accompanied by weaker coupling to external light (due in part to higher reflectivity of the dielectric-air interface), and therefore narrower transmission resonances of increasingly larger height. Original predictions of this effect (García de Abajo, 2002) have been recently corroborated by experiment using microwaves (García de Abajo et al., 2006).

An interesting situation is presented when localized modes like the ones described are mixed with extended lattice modes, like surface states underlying extraordinary optical transmission (García de Abajo et al., 2006; Ruan and Qiu, 2006). The interplay between both types of modes is illustrated in Fig. 12 through the zero-order transmittance of hole arrays filled with high-permittivity dielectric, calculated from the formalism presented in Sec. III.C.3. Incident light interacts with the cavity modes, giving rise to omnidirectional extraordinary transmission and invisibility behavior near the individual hole resonance (Borisov et al., 2005; García de Abajo, Gómez-Santos, et al., 2005). However, only p-polarized light couples to the $n=1, l=0$ lattice singularity of Fig. 4, which results in an avoided crossing of the hybridized modes [Fig. 12(a)]. Similar avoided crossings have been recently found in microwave experiments (Hibbins et al., 2006), confirming lattice surface modes and localized modes as two distinct mechanisms leading to enhanced 


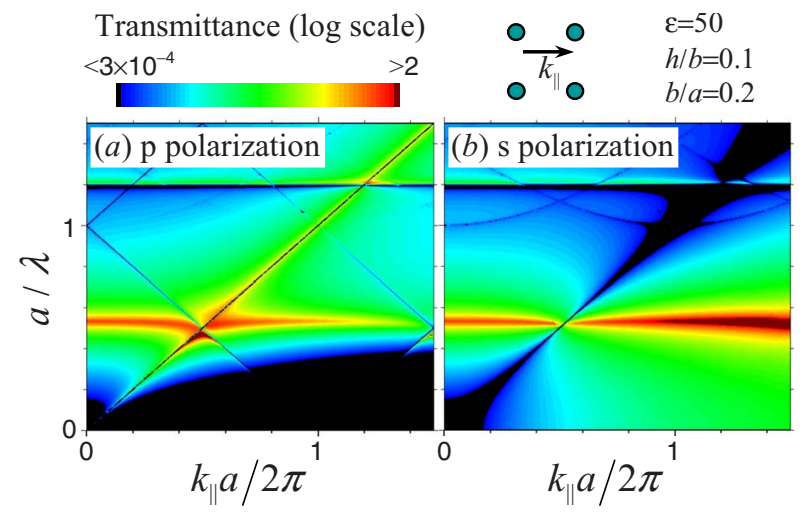

FIG. 12. (Color online) Interplay between localized (site) and extended (lattice) resonances. The contour plots show the zero-order beam transmittance of a square array of circular holes drilled in a perfect-conductor film and filled with dielectric material of permittivity $\epsilon=50$ as a function of parallel momentum $\mathbf{k}_{\|}$and wavelength $\lambda$. The orientation of $\mathbf{k}_{\|}$and the ratios between the hole radius $b$, the lattice constant $a$, and the film thickness $h$ are specified in the insets. The light is (a) $p$ polarized and (b) $s$ polarized. A transmission coefficient larger than 1 is only possible for evanescent waves below the light cone.

transmission. ${ }^{5,6}$ Notice that $s$-polarized light is immune to the $l=0$ lattice singularities of Fig. 4 , and this results in a reduced number of transmission features as compared to $p$ polarization, in qualitative agreement with experimental observations (Barnes et al., 2004).

Site resonances can occur in coaxial waveguides as well, via the so-called TEM mode, which does not have a cutoff in wavelength (Jackson, 1999). This led Roberts and McPhedran (1988) to theoretically explore the performance of periodic annular-hole arrays as band filters. More recently, Fan et al. (2005) measured the increased transmission of infrared light assisted by these modes. Similar coupling to localized TEM modes occurs as well in slits, as we discuss in Sec. III.F.

The type of interplay phenomenon that we are describing has been observed as well for localized and extended surface plasmons in the visible regime through the absorption features of porous metals, in which Mie modes of spherical cavities in otherwise planar surfaces display a rich structure of hybridization and avoided crossings (Kelf et al., 2005, 2006; Baumberg 2006; Teperik, Popov, Garcia de Abajo, Abdelsalam, et al., 2006; Teperik, Popov, Garcia de Abajo, Kelf, et al., 2006). The absorption can be even complete under attainable experimental conditions (Teperik et al., 2005), implying

\footnotetext{
${ }^{5}$ In a related context, avoided crossing of lattice modes are well known to occur in coinciding Wood anomalies (Stewart and Gallaway 1962).

${ }^{6}$ Incidentally, lattice modes are observed outside the light cone for $p$ polarization. The transmission outside that cone is defined as the squared-amplitude ratio of incident and transmitted evanescent waves at the exit and entrance surfaces of the film, respectively.
}

black-body-like emission according to Kirchhoff's laws of thermal radiation (Reif, 1965).

\section{F. Slit and cylinder arrays}

Although we have extracted conclusions for particles and holes from his works, Wood reported his anomalies for ruled gratings rather than 2D structures (Wood, 1902, Wood 1935). ${ }^{7}$ In fact, like gratings, cylinder and slit arrays exhibit lattice-resonance phenomena. But in contrast to holes, a single arbitrarily narrow slit in a perfect conductor supports at least one guided wave, the TEM mode (Jackson, 1999), which can couple to external $p$-polarized light (magnetic field parallel to the slit) giving rise to predicted (Takakura, 2001) and observed (Yang and Sambles, 2002) Fabry-Perot resonances in transmission (i.e., like the multiple peaks of the $\epsilon=50$ curve in Fig. 11). As a consequence, light passage through slit arrays can be assisted either by coupling to the TEM mode or by lattice resonances for $p$ polarization (Porto et al., 1999), leading to similar interplay between localized and extended resonances as discussed above (Marquier et al., 2005). Incidentally, the analogy with annular hole arrays is clear (see Sec. III.E).

We consider first a periodic array of parallel narrow cylinders, the axes of which define a single plane. Continuing with our tutorial approach, and focusing for simplicity on light incident with its electric field parallel to the cylinders, we note that Eqs. (2)-(4) are still applicable here, provided $\alpha_{E}$ and $\mathcal{G}^{0}$ are conveniently redefined. In particular, the polarizability has now dimensions of area rather than volume, and it is given, for instance, by $\alpha_{E}^{\mathrm{es}}=\pi b^{2}(\epsilon-1)$ for homogeneous cylinders of radius $b$ and permittivity $\epsilon$ (Bohren and Huffman, 1983), with the optical theorem now leading to $\operatorname{Im}\left\{1 / \alpha_{E}\right\}=-k^{2} / 4$. The relevant dipole-dipole interaction component is given by the Green's function of the Helmholtz equation in two dimensions, $\mathcal{G}^{0}=\left(i k^{2} / 4\right) H_{0}^{(1)}(k R)$, where $R$ is the distance measured in a plane perpendicular to the cylinders and $H_{0}^{(1)}$ is a Hankel function (Abramowitz and Stegun, 1972). Then, proceeding with the lattice sum $G\left(\mathbf{k}_{\|}\right)$analogous to Eq. (7), one finds a relation similar to Eq. (11) for the reflection coefficient of an array of lossless cylinders:

$$
r=\frac{-1}{1+(2 i a / k) \operatorname{Re}\left\{1 / \alpha_{E}-G(0)\right\}} .
$$

Under normal incidence ( $\left.k_{\|}=0\right), G$ is found to diverge as

$$
G(0) \approx \frac{\pi}{a^{2} \sqrt{2}} \frac{1}{\sqrt{\lambda / a-1}}
$$

for $\lambda \gtrsim a$, where $a$ is the lattice period. This is similar to particle arrays [see Eq. (13)], so that the main conclusions from our previous discussion of those arrays apply

\footnotetext{
${ }^{7}$ The reader is referred to the papers collected by Maystre (1993) for an exciting historical overview of twenty century milestones on gratings.
} 

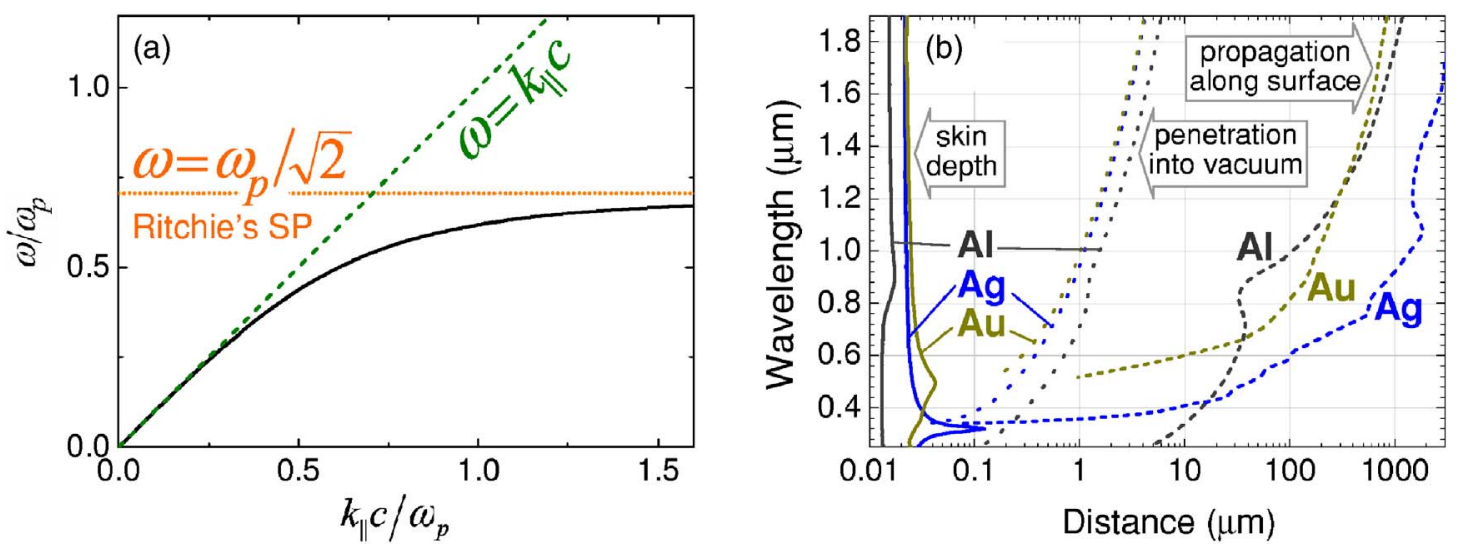

FIG. 13. (Color online) Summary of surface plasmon properties. (a) Surface plasmon dispersion relation for a Drude metal of bulk plasmon frequency $\omega_{p}$. (b) Extension of the plasmon field into the metal (skin depth), into the vacuum, and along the surface (propagation distance) for several metals, as obtained from measured optical constants (Johnson and Christy, 1972; Palik, 1985).

here as well, and more precisely the reflectivity can be made $100 \%$ for arbitrarily narrow or weakly scattering $(\epsilon \gtrsim 1)$ cylinders.

A complete analysis along these lines has been recently reported for all possible incident polarizations (Gómez-Medina et al., 2006; Laroche et al., 2006), suggesting that similar lattice resonances, somewhat less pronounced, are obtained for $\mathbf{E}^{\mathrm{ext}}$ perpendicular to the cylinders and with nonvanishing projection normal to the plane of the array. However, polarization components parallel to the plane and perpendicular to the cylinders cannot generate lattice resonances, because the interaction between distant dipoles aligned with their separation vector $\mathbf{R}$ decays as $1 / R^{3 / 2}$ in $2 \mathrm{D}$, which is insufficient to produce a divergence in $G{ }^{8}$

Finally, we establish a relation between cylinder arrays and slit arrays using arguments similar to those of Sec. III.C.1 for particle and hole arrays. More precisely, a slit array cut into a thin metal screen and illuminated with $\mathbf{E}^{\text {ext }}$ perpendicular to the apertures can be analyzed using the above results as applied to the Babinet-related stripe array (i.e., a periodic array of stripes cut in a planar thin screen) for $\mathbf{E}^{\text {ext }}$ parallel to the stripes. Under normal incidence, the required component of the polarizability reads $\alpha_{E} \approx-2 \pi / k^{2}[\ln (k b / 8)+\gamma+i \pi / 2]$, where $\gamma=0.57721$ is the Euler constant and $b \ll \lambda$ is the stripe width (van de Hulst, 1981). Interestingly, $\alpha_{E}$ diverges in the electrostatic limit, so that even a single narrowing slit will exhibit a divergent transmission cross section. This scenario can be traced back to the abovementioned site resonances produced by the TEM mode of slits in thick screens. As a consequence, the interaction between slits can be very large, resulting in strong redshifts of the transmission peaks relative to the Rayleigh condition.

\section{REAL METALS VS PERFECT CONDUCTORS}

Metals of finite conductivity show significant differences with respect to the perfect conductors considered

\footnotetext{
${ }^{8}$ This is because $\Sigma_{n=1}^{\infty} 1 / n^{3 / 2}$ is finite. See also Sec. III.B.
}

so far, the most remarkable of which is the existence of intrinsic surface-plasmon excitations. The basic understanding of these differences were laid out by Maystre (1972) in the context of diffraction gratings [see also McPhedran and Maystre (1974) and Maystre (1984)]. Next we examine the consequences for the interaction between particles and holes decorating metal surfaces.

\section{A. Surface plasmons}

Conduction electrons in metals behave like a plasma that is capable of sustaining collective oscillations known as plasmons (e.g., longitudinal bulk modes, signalled by the vanishing of the dielectric function). The existence of genuine surface plasmon oscillations was predicted by Ritchie (1957) and confirmed by electron energy-loss experiments (Powell and Swan, 1959). Since then, surface plasmons have developed into the rapidly growing field of plasmonics (Barnes et al., 2003; Ozbay, 2006; Zia et al., 2006) owing to their potential applicability to areas as diverse as biosensing (Schuster et al., 1993), signal processing through plasmonic circuits (Bozhevolnyi et al., 2006), or laser technology (Colombelli et al., 2003).

Planar surfaces possess translational invariance that provide plasmons with well-defined parallel momentum $k_{\|}$exceeding that of light outside the metal and thus becoming truly surface-bound modes. Their dispersion relation can be readily derived from the divergence of the Fresnel coefficients for $p$ polarization (surfacebound fields without external sources), leading to (Raether, 1988)

$$
k_{\|}^{\mathrm{SP}}=k \sqrt{\frac{\epsilon}{\epsilon+1}}
$$

for a metal-air interface. This surface plasmon dispersion relation is represented in Fig. 13(a) for a Drude metal described by Eq. (12). In the long $k_{\|}$limit, the surface plasmon frequency saturates to Ritchie's nonretarded plasmon (Ritchie, 1957).

Surface plasmons are characterized by three different length scales, as depicted in Fig. 13(b): their propagation distance along the surface $\left(\sim 1 / 2 \operatorname{Im}\left\{k_{\|}^{\mathrm{SP}}\right\}\right)$, their penetra- 
tion into the surrounding medium $\left(\sim 1 / 2 \operatorname{Im}\left\{k_{\perp}\right\}\right.$, where $k_{\perp}=-k / \sqrt{\epsilon+1}$ is the normal momentum), and their penetration into the metal (the skin depth $\sim 1 / 2 \operatorname{Im}\left\{-\epsilon k_{\perp}\right\}$ ). Interestingly, the interaction between plasmons on either sides of a thin film gives rise to two plasmon branches, as measured by electron microscopy (Vincent and Silcox, 1973; Pettit et al., 1975), one of which has been found to propagate along very long distances thanks to exclusion of the electric field from the metal (Sarid, 1981). Well-defined plasmons require $\operatorname{Im}\{\epsilon\}$ $\ll \operatorname{Re}\{-\epsilon\}$, but similar long-range surface-exciton polaritons exist in thin films for $\operatorname{Im}\{\epsilon\} \gg|\operatorname{Re}\{\epsilon\}|$ (Yang et al., 1990).

Features in metal surfaces produce scattering of plasmons in a similar way as light is dispersed by particles. This is actually a way to couple externally incident light to plasmons, for instance, using gratings (Ritchie et al., 1968; Loewen et al., 1984). We find a demonstration of these ideas in the observation of surface-plasmon bands for periodic surface decoration (Stewart and Gallaway, 1962; Ritchie et al., 1968; Kitson et al., 1996), and in the reflection of surface plasmons at point scatterers arranged as parabolic mirrors (Nomura et al., 2005). Similarly, holes perforating films have a strong influence on surface plasmons, which play an important role in their optical transmission (Ghaemi et al., 1998). However, in the perfect-conductor limit, with $|\epsilon| \rightarrow \infty$, Eq. (25) yields $k_{\|}^{\mathrm{SP}}=k$, with zero skin depth and infinite penetration into the vacuum, that is, there are no longer surface-bound modes. In the following we explore the transition between plasmonic and perfect-conductor regimes, in an attempt to clarify contradictory statements regarding the role of surface plasmons to enhance (Schröter and Heitmann, 1998) or to suppress (Cao and Lalanne, 2002) extraordinary optical transmission in striped thin films, or the debate opened by the explanation of recent experiments dealing with the interaction between a slit and a groove (García-Vidal et al., 2006; Gay et al., 2006; Lalanne and Hugonin, 2006).

\section{B. Polarization schemes}

The condition that parallel electric dipoles and perpendicular magnetic dipoles are excluded from perfectconductor surfaces (see Fig. 10) is relaxed in metals of finite conductivity. Polarization charges in a hole for instance can lead to a net parallel electric dipole in a thin metallic film (Rindzevicius et al., 2007).

In order to illustrate this concept, we consider in Fig. 14 the effective polarizability of a silver spherical particle in front of a silver surface for a constant ratio of the radius to the wavelength, $b / \lambda=0.1$. We observe an electric Mie mode (Mie, 1908) in the visible, accompanied by negligible magnetic response. However, the metal behaves increasingly closer to a perfect conductor at longer wavelengths, so that currents compete eventually with polarization, thus displaying magnetic polarizability that becomes $\alpha_{M}=-b^{3} / 2$ for an isolated perfectconductor sphere in the long-wavelength limit (Jackson,

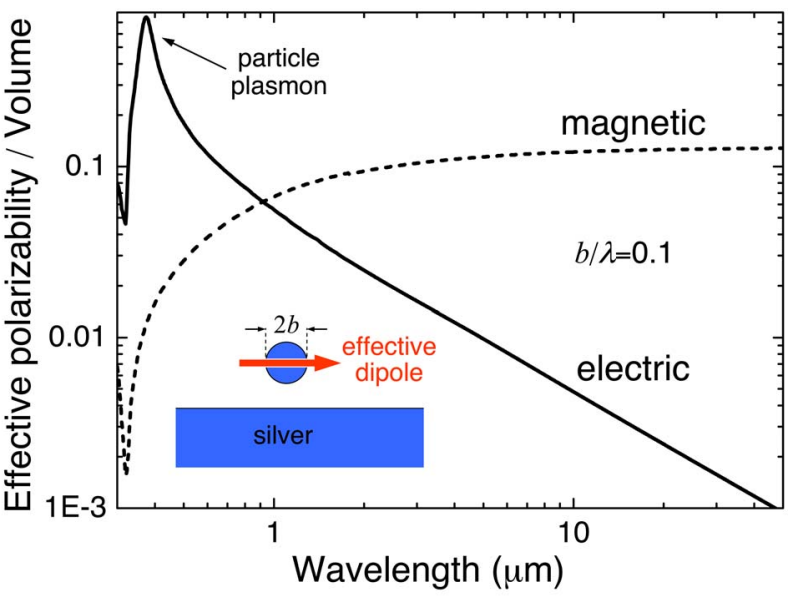

FIG. 14. (Color online) Effective polarization strength of a silver sphere near a silver planar surface. The sphere radius is a tenth of the wavelength. The polarization is normalized to the sphere volume. The dielectric function of silver is from Johnson and Christy, 1972.

1999), to be compared with the electric polarizability $\alpha_{E}=b^{3}$. Nevertheless, the latter is quenched by proximity of the metal flat surface under normal-incidence illumination conditions. The onset of magnetic response occurs when the particle becomes large compared to the skin depth $\sim 20 \mathrm{~nm}$ [see Fig. 13(b)]. These results follow from dipolar Mie scattering, conveniently corrected by surface reflection coefficients, which qualitatively describe the polarizability strength of the coupled particlesurface system.

This has important consequences for understanding patterned surfaces and hole arrays. Electric dipoles dominate the response of features smaller than the skin depth, whereas magnetic dipoles can be significant for larger sizes, and only parallel electric dipoles and perpendicular magnetic dipoles survive in the limit of negligible skin depth. We are of course restricting our discussion to particles or apertures that are small compared to the wavelength, but these conclusions can be generalized to higher-order multipoles for bigger features.

\section{Dipole-dipole interaction}

New dipole orientations and the presence of surface plasmons in real metals demand that we revisit the interaction between features in tailored surfaces. In particular, the dipolar field in free space, which decays away from the source as

$$
\mathcal{G}^{0} \sim \frac{e^{i k R}}{R}
$$

and governs the interaction between small features in perfect-conductor surfaces (see Sec. III.A), must be 


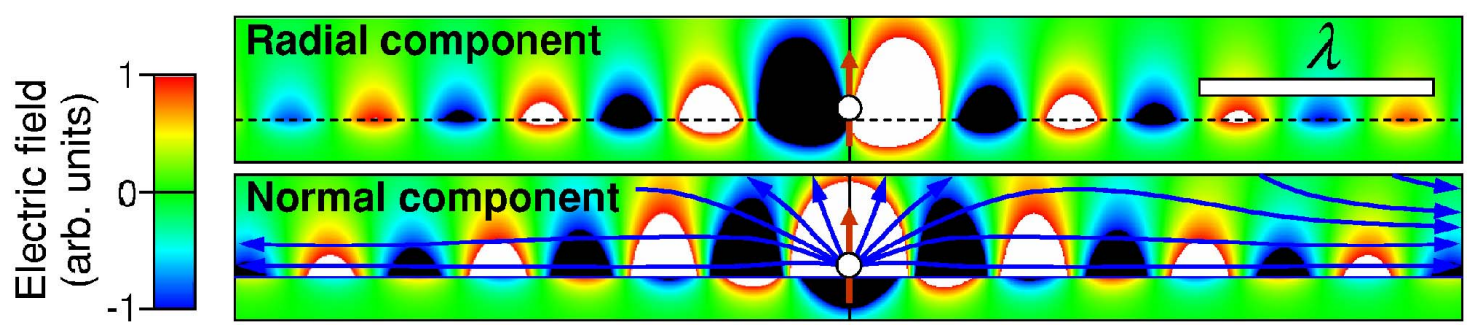

FIG. 15. (Color online) Instantaneous induced electric field set up by a perpendicular electric dipole (see vertical arrows) sitting at a distance $\lambda / 20$ from the surface of a metal described by Eq. (12) with $\omega_{p}=15 \mathrm{eV}$ and damping $\eta=0.6 \mathrm{eV}$ (typical of Al) at frequency $\omega=\omega_{p} / 2$. The electric-field component parallel to the surface (this is radial with respect to the position of the dipole) and the component along the surface normal are represented separately. Poynting vector flow lines are superimposed on the plot of the normal component.

supplemented by reflected fields near real metals, leading to an interaction tensor of the form ${ }^{9}$

$$
\mathcal{G}=\mathcal{G}^{0}+\mathcal{G}^{r} .
$$

As a result, light impinging on a hole can couple to circular surface-plasmon waves (Wannemacher, 2001; Yin et al., 2004; Chang et al., 2005; Popov et al., 2005), whose field strength shows a rather different decay dependence with distance as

$$
\mathcal{G} \sim \frac{e^{i k_{\|}^{\mathrm{SP}} R}}{\sqrt{R}}
$$

This expression is consistent with energy flux conservation for any surface-bound mode, ${ }^{10}$ with dissipation described through the imaginary part of $k_{\|}^{\mathrm{SP}}$. The slow drop of Eq. (27) with distance compared to Eq. (26) can explain the observed enhancement of the interaction between small particles in plasmonic metals (Stuart and Hall, 1998), and is illustrated in Fig. 15, showing the field produced by a dipole near a metallic surface calculated from an extension of our tutorial approach formalism presented below.

The interaction between pairs of electric and magnetic dipoles near a metal surface is analyzed in Fig. 16(a) for all possible orientations except perpendicular magnetic dipoles, which are forbidden in perfect conductors and should take small values in real metals. Moreover, symmetry forbids the interaction of all other pairs that are not shown in the figure. For surface features inducing

\footnotetext{
${ }^{9}$ In the perfect conductor limit, $\mathcal{G}^{r}$ has the effect of canceling exactly some of the components of $\mathcal{G}^{0}$ and multiplying by 2 the remaining ones. In fact, that factor of 2 was absorbed in the polarizabilities considered in Sec. III. The self-consistent equations for interaction among holes presented in that section can be still applied to plasmonic metals, with the dipole-dipole interaction $\mathcal{G}$ now approaching $2 \mathcal{G}^{0}$ (and the hole polarizability going to half the value of Fig. 7) in the perfect-conductor limit.

${ }^{10}$ The Poynting vector produced by a dipole when fields are propagated by Eq. (27) dies off as $1 / R$, if we neglect the attenuation produced by $\operatorname{Im}\left\{k_{\|}^{\mathrm{SP}}\right\}$. The integral of the radial Poynting vector over a circle of radius $R$ centered around the dipole and lying on the surface is independent of $R$, so that the photon flux is conserved, indicating surface-bound propagation.
}
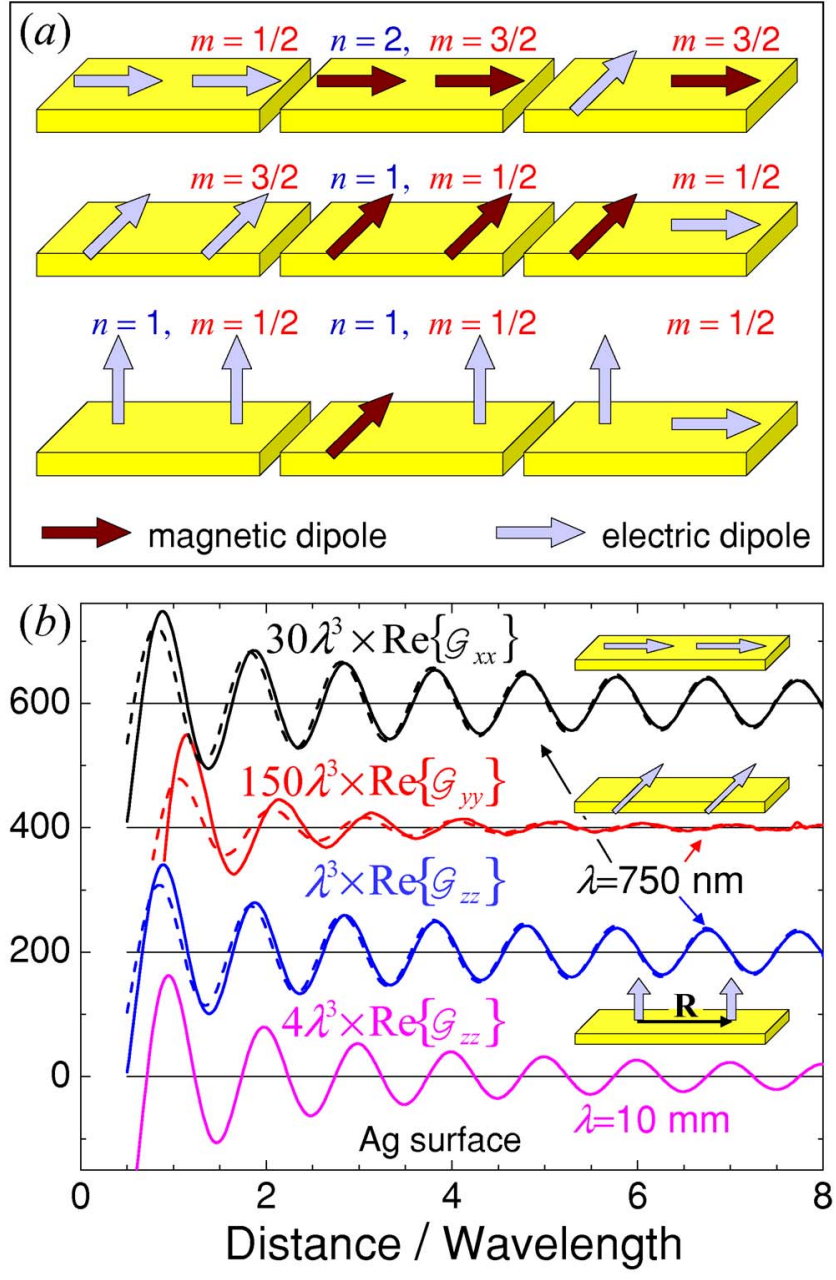

FIG. 16. (Color online) Dipole-dipole interaction in metallic surfaces. (a) Schematic representation of the scaling of dipoledipole interactions for electric and magnetic dipoles with respect to their separation $R$ near a metallic surface. The interaction decays as $\exp (i k R) / R^{n}$ near a perfect conductor or as $\exp \left(i k_{\|}^{\mathrm{SP}} R\right) / R^{m}$ near a metal with a dominant surface plasmon (see insets for values of the exponents $n$ and $m$ ). (b) Dipoledipole interaction near a silver surface at a wavelength of $750 \mathrm{~nm}$ (three upper solid curves) as compared with the plasmon-pole approximation (three upper dashed curves, see text). We also show the interaction at a wavelength of $10 \mathrm{~mm}$ (lower curve, perfect-conductor limit). The dipole-dipole separation vector $\mathbf{R}$ is taken along $\hat{\mathbf{x}}$. 
electric dipoles under normal incidence in a plasmonic metal (see Fig. 14), the dominant interactions originate in electric-dipole pairs aligned with their separation vector $\mathbf{R}$ (see Fig. 16), quite different from perfect conductors, which are governed by magnetic dipoles perpendicular to $\mathbf{R}$. However, the latter can contribute in plasmonic materials as well for large features compared to the skin depth, as discussed in Sec. IV.B. As a rule of thumb, mutual dipole orientations that lead to the longrange interaction dependence given by Eq. (27) are compatible with nonvanishing surface-plasmon field components emanating from those dipoles (i.e., plasmons with $m=0$ azimuthal symmetry for normal electric dipoles, like in Fig. 15, or $m= \pm 1$ for parallel dipoles).

The interaction between dipoles in front of a planar surface admits a representation in parallel momentum space similar to Eq. (5), but involving now the Fresnel reflection coefficients for $s$ and $p$ polarization (Weyl, 1919; Blanco and García de Abajo, 2004), $r_{s}=\left(k_{z}\right.$ $\left.-k_{z}^{\prime}\right) /\left(k_{z}+k_{z}^{\prime}\right)$ and $r_{p}=\left(\epsilon k_{z}-k_{z}^{\prime}\right) /\left(\epsilon k_{z}+k_{z}^{\prime}\right)$, respectively (Jackson, 1999), where $k_{z}=\sqrt{k^{2}-Q^{2}}$ and $k_{z}^{\prime}=\sqrt{k^{2} \epsilon-Q^{2}}$. In particular, for electric dipoles parallel to the surface $x$ direction, one finds (Weyl, 1919; Ford and Weber, 1984)

$$
\mathcal{G}_{x x}^{r}=\frac{i}{2 \pi} \int \frac{d^{2} \mathbf{Q}}{k_{z} Q^{2}} e^{i\left(\mathbf{Q} \cdot \mathbf{R}+k_{z}|z|\right)}\left[k^{2} Q_{y}^{2} r_{s}-k_{z}^{2} Q_{x}^{2} r_{p}\right],
$$

where $z$ is the sum of distances from the dipoles to the surface, and we are interested in the $z \rightarrow 0$ limit. This expression is general and leads to $\mathcal{G}_{x x}=0$ in perfect conductors, for which $r_{p}=-r_{s}=1$.

The strong surface-plasmon-mediated interaction described by Eq. (27) arises from the pole of the Fresnel coefficient $r_{p}$ at $Q=k_{\|}^{\mathrm{SP}}$, which admits the Laurent expansion (Ford and Weber, 1984)

$$
r_{p} \approx \frac{2 B k}{Q-k_{\|}^{\mathrm{SP}}},
$$

with

$$
B=[\epsilon /(1+\epsilon)]^{3 / 2} /(1-\epsilon) .
$$

Performing asymptotic analysis for large $R$ and retaining only the contribution from this pole in the integral of Eq. (28) (plasmon-pole approximation; see Ford and Weber, 1984), we obtain ${ }^{11}$

\footnotetext{
${ }^{11}$ It should be noted that the asymptotic behavior of $\mathcal{G}^{0}$ [see Eq. (26)] comes from the stationary phase of the exponential in the integral of Eq. (5). This contribution is canceled exactly by Eq. (28), in which $r_{p}=r_{s}=-1$ at grazing incidence (i.e., for $Q$ $=k$ ). Therefore the only relevant contribution to $\mathcal{G}$ for large $R$ originates in the plasmon pole of $\mathcal{G}^{r}$.
}

$$
\begin{aligned}
\mathcal{G}_{x x} & \approx-\frac{\pi k^{3} B \sqrt{\epsilon}}{\epsilon+1}\left[H_{0}^{(1)}\left(k_{\|}^{\mathrm{SP}} R\right)+H_{2}^{(1)}\left(k_{\|}^{\mathrm{SP}} R\right) \frac{\left(y^{2}-x^{2}\right)}{R^{2}}\right] \\
& \approx \frac{-2 k^{3} B}{\epsilon+1} \sqrt{\frac{2 \pi \epsilon}{i k_{\|}^{\mathrm{SP}}}} \frac{x^{2}}{R^{2}} \frac{e^{i k_{\|}^{\mathrm{SP}} R}}{\sqrt{R}},
\end{aligned}
$$

where the second approximation comes from the asymptotic behavior of Hankel functions for large arguments (Abramowitz and Stegun, 1972), so that one obtains the result anticipated in Eq. (27). The above expression in terms of Hankel functions is compared with the direct numerical evaluation of Eq. (28), and similar expressions for other dipole orientations, in Fig. 16(b). The agreement at $\lambda=750 \mathrm{~nm}$ is excellent for $R \gtrsim \lambda$, indicating that lattice resonances in an array will be dominated by surface plasmons at that wavelength. Figure 16 also illustrates a much faster decay of $\mathcal{G}_{y y}$ as $1 / R^{3 / 2}$ for electric dipoles oriented orthogonal to $\mathbf{R}$ and parallel to the surface, and as $1 / R$ for normal electric dipoles in the perfect-conductor limit.

\section{Discrepancies in lattice resonances and enhanced transmission}

The dissimilar behavior of plasmonic metals and perfect conductors discussed leads to qualitative differences in extraordinary optical transmission, arising in part from the $1 /\left(Q-k_{\|}^{\mathrm{SP}}\right)$ dominant pole of the interhole interaction in momentum space [see Eqs. (28)-(30)].

Considering for simplicity a square array under normal incidence, we analyze the lattice sum in a real metal [i.e., Eq. (4) with $\mathcal{G}$ substituted for $\mathcal{G}^{0}$ ] following the procedure that led to Eq. (7), but starting now from Eqs. (6) and (28). In a diffrationless array, there are two identical singular terms in the corresponding sum over reciprocallattice vectors, leading to

$$
G_{x x}^{\mathrm{EE}} \approx C\left(\frac{4 \pi}{a \lambda}\right)^{2} \frac{\lambda_{\mathrm{SP}}}{\lambda_{\mathrm{SP}} / a-1}
$$

for $\operatorname{Re}\left\{\lambda_{\mathrm{SP}}\right\} \gtrsim a$, where $\lambda_{\mathrm{SP}}=2 \pi / k_{\|}^{\mathrm{SP}}$ is the surfaceplasmon wavelength and $C=i B / \sqrt{\epsilon+1}$. We have explicitly indicated with superscripts that $G_{x x}^{\mathrm{EE}}$ describes the interaction between electric dipoles $(E)$, which can coexist with parallel magnetic dipoles $(M)$ (see Fig. 16). The remaining relevant lattice sums are $G_{y y}^{\mathrm{MM}} \approx-(\epsilon+1) G_{x x}^{\mathrm{EE}}$ and $G_{x y}^{\mathrm{EM}}=-G_{y x}^{\mathrm{ME}} \approx \sqrt{\epsilon+1} G_{x x}^{\mathrm{EE}}$. Now, the formalism presented in Sec. III can be easily extended to patterned surfaces and hole arrays in real metals using these expressions of the lattice sums rather than those for perfect conductors (see footnote 9). ${ }^{12}$

In the polaritonic regime of surface plasmons, in which their dispersion relation approaches the light line (see Fig. 13), $|\epsilon|$ is large and the dominant lattice sum

\footnotetext{
${ }^{12}$ Our analysis can be applied to metals embedded in a dielectric of refraction index $n$ simply by using the reduced wavelength $\lambda / n$ everywhere instead of $\lambda$ and by interpreting $\epsilon$ as the ratio of permittivities in the metal and in the dielectric.
} 


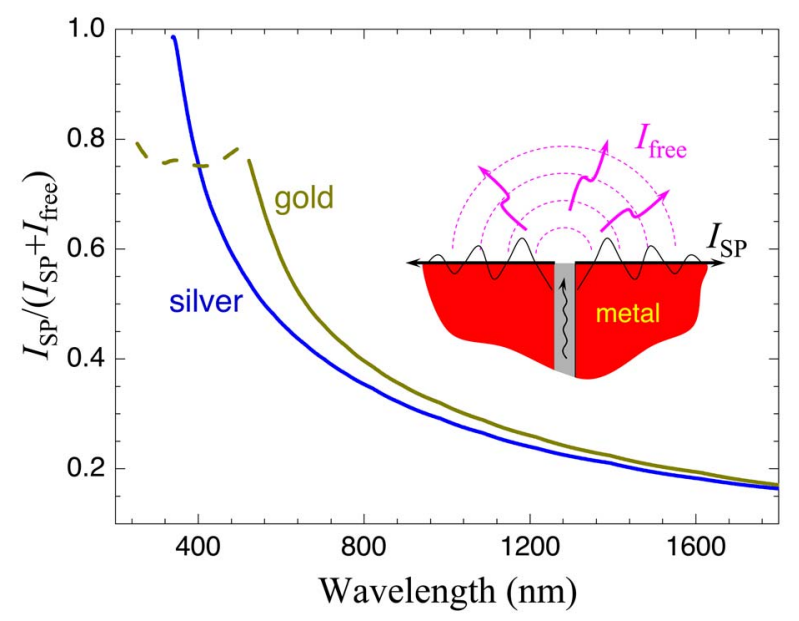

FIG. 17. (Color online) Relation between the power radiated after transmission through a deep subwavelength hole $\left(I_{\text {free }}\right)$ and the power emanating as surface plasmons $\left(I_{\mathrm{SP}}\right)$ for gold and silver, derived in the small-hole limit. [We obtain the emitted light intensity from integration of the radial Poynting vector corresponding to the field of Eq. (28) plus the dipole direct field over the upper hemisphere, far from the hole. The SP intensity comes from the Poynting vector normal to a cylindrical surface centered at the hole, with the field given by Eq. (30).] The metal dielectric function is from Johnson and Christy, 1972.

scales as $G_{y y}^{\mathrm{MM}} \sim 1 / \sqrt{-\epsilon}$ in the plasmon-pole approximation, so that for sufficiently high $|\epsilon|$ the perfectconductor limit of Eq. (13) dominates over the plasmon.

A descriptive example of the transition from plasmonic to perfect-conductor behavior is given in Fig. 17, in which the energy released by an electric dipole sitting near and parallel to a surface is divided into plasmon launching $\left(I_{\mathrm{SP}}\right)$ and emitted light $\left(I_{\text {free }}\right)$. This relates to the question, which of the two mechanisms (plasmons or propagating radiation) produces stronger interaction with a nearby surface feature. Plasmon launching dominates near the electrostatic plasmon, reaching an efficiency close to $100 \%$ in silver. As the wavelength advances towards to infrared, the plasmon is less bound to the surface and has weaker coupling to our dipole. As an example, when light emits out of a narrow hole after being guided through a TE mode (e.g., in a circular hole infiltrated with a dielectric of refraction index $n \gg 1$ and for $\lambda / n \lesssim 3.4 b$ ), the equivalent dipole describing the hole lies parallel to the surface. This is the situation depicted in the inset of Fig. 17.

A more explicit comparison of discrepancies between both metallic regimes for holes is given in Fig. 18, which shows the lattice sum $G_{y y}^{\mathrm{MM}}$ for parallel magnetic dipoles [obtained by summing Eq. (28) for gold, with the expression in square brackets replaced by $k^{2} Q_{x}^{2} r_{p}-k_{z}^{2} Q_{y}^{2} r_{s}$ ], together with a geometrical construction like in Fig. 6, applied now to two different aperture sizes. It should be noted that the exact calculation (solid curves) compares well with analytical expressions [symbols, obtained from Eq. (13) for the perfect conductor and from Eq. (31) for the plasmonic metal, which needs to be multiplied by

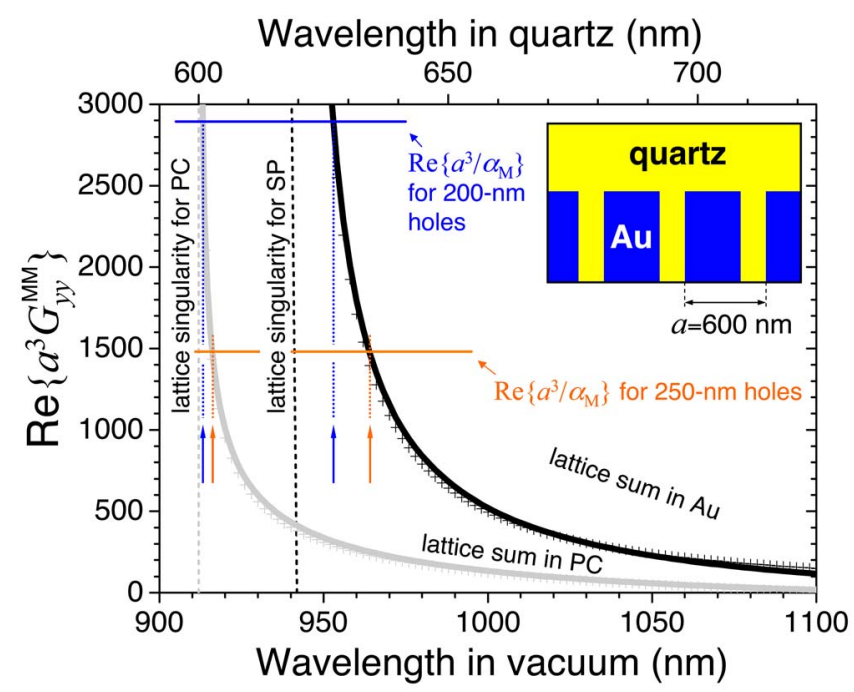

FIG. 18. (Color online) Lattice sums and lattice resonances in a square array of holes drilled in gold vs a perfect conductor. The real part of the exact lattice sum for interaction of parallel magnetic $(M)$ dipoles is shown for gold (black curve) and for a perfect conductor (PC, gray curve), as compared to analytical approximate expressions (symbols). The Rayleigh condition for a period $a=600 \mathrm{~nm}$ is indicated by gray and black vertical dashed lines for light in the dielectric $(\lambda / n=a)$ and for surface plasmons $\left(\lambda_{\mathrm{SP}}=a\right)$, respectively. Changes in the inverse magnetic polarizability of circular holes of different size [horizontal lines, as obtained from Fig. 7(b) (see footnote 9)], lead to different wavelengths of the lattice surface modes, as indicated by vertical arrows for the condition that the real part of the denominator of Eq. (3) be zero.

$-(\epsilon+1)$ in order to apply it to magnetic rather than electric dipoles]. The lattice sum singularity in perforated gold takes place to the red as compared to the perfectconductor case, because the surface-plasmon wavelength is shorter than the light wavelength in the surrounding dielectric. Moreover, the lattice sum diverges as $1 / \sqrt{\lambda / n-a}$ and $1 /\left(\lambda_{\mathrm{SP}}-a\right)$ in perfect conductors and plasmonic metals, respectively, according to Eqs. (13) and (31), thus leading to different dependence of the position of the lattice surface resonance on hole size (see points of intersection with horizontal lines in Fig. 18); the lattice resonance is further away from the interaction sum singularity (and a given change in hole diameter produces larger peak shift) in the plasmonic case considered in the figure.

The crossover between both types of behavior is explored in Fig. 19 through the absorbance of (i) a silverparticle array in silica, (ii) the same array near a silversilica interface, and (iii) an array of silica inclusions right underneath the metal-dielectric interface. We have done these calculations using a layer KKR method to solve Maxwell's equations (Stefanou et al., 1998, 2000). In the case (i) a maximum in absorption occurs near the Rayleigh condition for light propagating in silica (i.e., $\lambda / n$ $=a$ ), whereas case (iii) shows a single maximum shifted to the right of the Rayleigh condition for the planar interface plasmon $\left(\lambda_{\mathrm{SP}}=a\right)$ (Ghaemi et al., 1998). The con- 


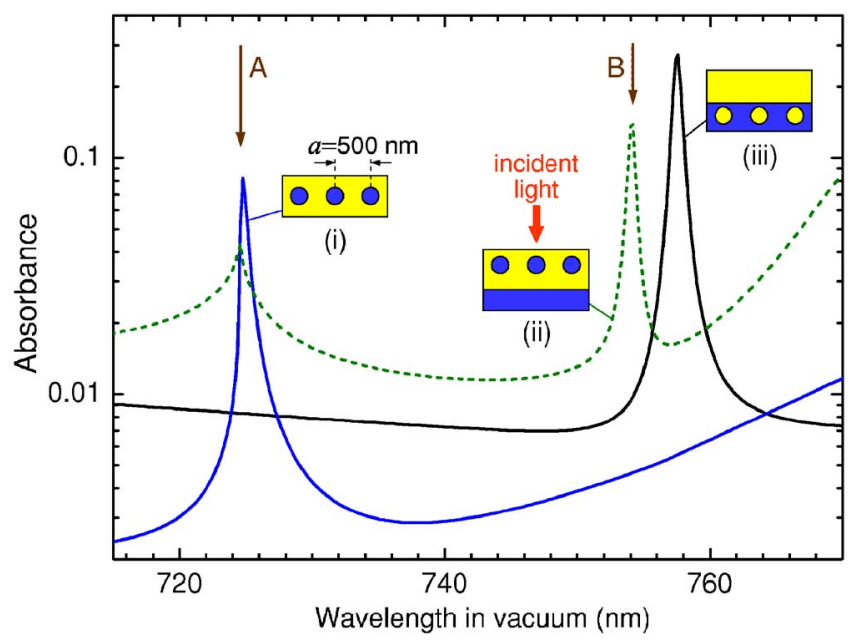

FIG. 19. (Color online) Normal-incidence absorbance of (i) a silver particle array embedded in silica (refraction index $n$ =1.45), (ii) the same array near a planar silver-silica interface, and (iii) an array of silica inclusions buried in silver below a silver-silica interface. All particles are spheres of $200 \mathrm{~nm}$ in diameter. The arrays have square symmetry with lattice constant $a=500 \mathrm{~nm}$. The distance from the sphere surfaces to the planar interface is $10 \mathrm{~nm}$ in the buried silica particles and $900 \mathrm{~nm}$ for the silver particles. The Rayleigh conditions for the reduced wavelength of light in the silica $(\lambda / n=a)$ and for the wavelength of the silver-silica interface plasmon $\left(\lambda_{\mathrm{SP}}=a\right)$ are indicated by arrows $A$ and $B$, respectively.

clusion is that plasmons are mediating the interaction among the dielectric inclusions, with no signature of any anomaly near $\lambda / n=a$ whatsoever. An intermediate situation is encountered in case (ii), showing features near the two types of Rayleigh conditions.

It should be noted that $\lambda_{\mathrm{SP}}$ has an imaginary part arising from absorption, and although it is small for noble metals, in which plasmons can travel long distances along the surface, as shown in Fig. 13(b), we find that Eq. (31) does not describe a true divergence, but rather a Lorentzian of finite width. This affects the height of the transmission maxima, below $100 \%$ in lossy metals. Furthermore, apertures perforated in metals of finite conductivity will appear to be wider with the skin depth effect, and their effective polarizability must be lossy.

Without entering into further considerations regarding how finite conductivity affects the hole polarizability, we point out that the wavelength at which the noted intersection takes place in Fig. 18 (i.e., the wavelength of the lattice surface-bound mode) is in agreement with the transmission peaks measured by Krishnan et al. (2001) and reproduced in Fig. 1(a). The vertical arrows in Fig. 1 indicate the predicted positions of the transmission maxima, obtained by increasing the hole size by the skin depth to an effective diameter of $250 \mathrm{~nm}$. This agreement is remarkable, given our neglect of higher-order multipolar terms in the hole polarization. The shift with respect to the Rayleigh condition for surface plasmons (vertical solid lines in Fig. 1) is significant, triggered by large, plasmon-mediated interaction between apertures. Similar conclusions can be drawn for the silver film of
Fig. 1(b), in which the results from the above analytical model are shown as dashed curves (divided by a factor of 5). Only magnetic dipoles are taken into account, with the hole polarizability calculated for a perfect conductor. The transmittance is obtained from Eq. (19) with $G_{x x}$ replaced by its plasmonic counterpart $G_{y y}^{\mathrm{MM}}$. Although the Rayleigh condition for plasmons (solid vertical lines in Fig. 1) agrees only with the transmission minima in silver (presumably because gold is more dissipative in this spectral region, so that the polarizability of the holes requires a more realistic description including absorption), comparison with experiment is excellent, given the simplicity of the analytical model, which should become exact in the limit of small scattering features (e.g., for nanoparticle arrays on a metal substrate).

\section{CONCLUSION}

Light scattering in planar periodic systems gives rise to resonant phenomena that have common origins in particle and hole arrays, both for reflection and for transmission. Namely, (i) the interaction between lattice sites shows a divergent behavior when a diffracted beam becomes grazing (Lord Rayleigh, 1907), producing a minimum in both the reflectivity of particle arrays and the transmission of hole arrays; (ii) a lattice resonance can be established at a wavelength to the red of this condition, leading to maxima in both the reflectivity of particle arrays and the transmission of hole arrays; (iii) these effects have the same origin as Wood's anomalies (Wood, 1935) and can be described in the language of Fano line shapes (Fano, 1961); (iv) the noted lattice resonance persists for incident evanescent light, with the reflectivity's becoming infinite in nondissipative systems (e.g., patterned perfect conductors, but also patterned dielectrics), thus defining truly surface-bound states (Ulrich and Tacke, 1972; Pendry et al., 2004; García de Abajo and Sáenz 2005; Hibbins et al., 2005); (v) these extended lattice resonances mix strongly with other modes localized at specific sites, like those created by nanoparticle and nanovoid plasmons (Kelf et al., 2006; Teperik, Popov, García de Abajo, Abdelsalam, et al., 2006); (vi) for metals with well-defined surface plasmons, the interaction between holes or particles in the vicinity of the surface is mediated by these excitations, so that we have to reformulate the condition of a diffracted beam's becoming grazing using the surface plasmon wavelength rather than the incoming or transmitted light wavelength.

We have shown that particle arrays and hole patterns in perfect conductors share in common the asymptotic form of their interaction, summarized by Eq. (26), which produces singularities at the Rayleigh condition when summed over the lattice, for instance, for $\lambda=a$ under normal incidence on square arrays, and gives rise to surface states at slightly larger wavelengths. However, the plasmon-mediated interaction in noble metals is more intense, as shown in Eq. (27), thus producing sharper divergences and stronger collective interaction. In this case, singularities occur at the band-folded plasmon 
lines (e.g., when $\lambda_{\mathrm{SP}}=a$ under normal incidence on square arrays), and the lattice surface-bound states (i.e., plasmons of the patterned metal) exist again to the red with respect to those lines.

All these effects have been described here within a common approach based upon interacting dipoles that is not only able to explain the observed effects; its simplicity has allowed us to extract some surprising conclusions. One of them is that arbitrarily weak scatterers forming a periodic structure and made of nondissipative materials can also produce intense lattice resonances: given an array of arbitrarily small particles of positive polarizability, it is always possible to find a wavelength (close to the period for square symmetry and normal incidence) at which light is totally reflected; accordingly, it is possible to obtain full transmission through holes however narrow, drilled in arbitrarily thick perfect-conductor films.

Interestingly, the lattice periodicity alone determines the magnitude of the induced dipoles needed to produce complete reflection by small particles or total transmission through narrow holes. Moreover, the polarizability scales with the cube of the hole or particle diameter. Combining these two statements, we find that the selfconsistent electric field acting on particles or apertures under such resonant conditions increases when they shrink and can reach extremely high values only limited by absorption and lattice imperfections, thus opening new possibilities for applications in nonlinear all-optical switching and biosensing.

The simplicity and power of the model presented here will find application to explain many other effects related to light scattering in planar periodic systems and can be inspiring for devising new phenomena.

\section{ACKNOWLEDGMENTS}

The author wants to thank J. J. Baumberg, A. G. Borisov, G. Gómez-Santos, C. López, F. Meseguer, J. B. Pendry, V. V. Popov, J. J. Sáenz, S. V. Shabanov, T. V. Teperik, and N. I. Zheludev for many enjoyable and stimulating discussions. This work was supported in part by the Spanish MEC (Contract No. NAN2004-08843C05-05) and by the EU (SPANS STREP STRP-016881 and Metamorphose NoE NMP3-CT-2004-500252).

\section{REFERENCES}

Abramowitz, M., and I. A. Stegun, 1972, Handbook of Mathematical Functions (Dover, New York).

Akahane, Y., T. Asano, B.-S. Song, and S. Noda, 2003, "High-Q photonic nanocavity in a two-dimensional photonic crystal," Nature (London) 425, 944-947.

Altewischer, E., M. P. van Exter, and J. P. Woerdman, 2002, "Plasmon-assisted transmission of entangled photons," Nature (London) 418, 304-306.

Ashcroft, N. W., and N. D. Mermin, 1976, Solid State Physics (Harcourt College Publishers, New York).

Atay, T., J.-H. Song, and A. V. Nurmikko, 2004, "Strongly interacting plasmon nanoparticle pairs: from dipole-dipole interaction to conductively coupled regime," Nano Lett. 4,
1627-1631.

Baida, F. I., and D. Van Labeke, 2002, "Light transmission by subwavelength annular aperture arrays in metallic films," Opt. Commun. 209, 17-22.

Barlow, H. M., 1958, "Surface waves," Proc. IRE 46, 14131417.

Barnes, W., and R. Sambles, 2004, "Only skin deep," Science 305, 785-786.

Barnes, W. L., A. Dereux, and T. W. Ebbesen, 2003, "Surface plasmon subwavelength optics," Nature (London) 424, 824830.

Barnes, W. L., W. A. Murray, J. Dintinger, E. Devaux, and T. W. Ebbesen, 2004, "Surface plasmon polaritons and their role in the enhanced transmission of light through periodic arrays of subwavelength holes in a metal film," Phys. Rev. Lett. 92, 107401.

Baumberg, J. J., 2006, "Breaking the mould: Casting on the nanometre scale," Nat. Mater. 5, 2-5.

Bethe, H. A., 1944, "Theory of diffraction by small holes," Phys. Rev. 66, 163-182.

Blanco, L. A., and F. J. García de Abajo, 2004, "Spontaneous light emission in complex nanostructures," Phys. Rev. B 69, 205414.

Bohren, C. F., and D. R. Huffman, 1983, Absorption and Scattering of Light by Small Particles (Wiley-Interscience, New York).

Borisov, A. G., F. J. García de Abajo, and S. V. Shabanov, 2005, "Role of electromagnetic trapped modes in extraordinary transmission in nanostructured materials," Phys. Rev. B 71, 075408.

Born, M., and E. Wolf, 1999, Principles of Optics: Electromagnetic Theory of Propagation, Interference and Diffraction of Light (Cambridge University Press, Cambridge, England).

Bouwkamp, C. J., 1954, “Diffraction theory,” Rep. Prog. Phys. 17, 35-100.

Bozhevolnyi, S. I., V. S. Volkov, E. Devaux, J.-Y. Laluet, and T. W. Ebbesen, 2006, "Channel plasmon subwavelength waveguide components including interferometers and ring resonators," Nature (London) 440, 508-511.

Bravo-Abad, J., F. J. García-Vidal, and L. Martín-Moreno, 2004, "Resonant transmission of light through finite chains of subwavelength holes in a metallic film," Phys. Rev. Lett. 93, 227401.

Cao, H., and A. Nahata, 2004, "Resonantly enhanced transmission of terahertz radiation through a periodic array of subwavelength apertures," Opt. Express 12, 1004-1010.

Cao, Q., and P. Lalanne, 2002, "Negative role of surface plasmons in the transmission of metallic gratings with very narrow slits," Phys. Rev. Lett. 88, 057403.

Chang, C.-W., A. K. Sarychev, and V. M. Shalaev, 2006, "Light diffraction by a subwavelength circular aperture," Laser Phys. Lett. 2, 351-355.

Chang, S.-H., S. K. Gray, and G. C. Schatz, 2005, "Surface plasmon generation and light transmission by isolated nanoholes and arrays of nanoholes in thin metal films," Opt. Express 13, 3150-3165.

Chen, C. C., 1971, "Diffraction of electromagnetic waves by a conducting screen perforated periodically with circular holes," IEEE Trans. Microwave Theory Tech. 19, 475-481.

Collin, R. E., and W. H. Eggimann, 1961, "Dynamic interaction fields in a two-dimensional lattice," IRE Trans. Microwave Theory Tech. 9, 110-115.

Colombelli, R., K. Srinivasan, M. Troccoli, O. Painter, C. F. 
Gmachl, D. M. Tennant, A. M. Sergent, D. L. Sivco, A. Y. Cho, and F. Capasso, 2003, "Quantum-cascade surfaceemitting photonic crystal laser," Science 302, 1374-1377.

Cwik, T., R. Mittra, K. C. Lang, and T. K. Wu, 1987, "Frequency selective screens," IEEE Trans. Antennas Propag. Soc. Newsl. 29, 5-10.

Dawes, D. H., R. C. McPhedran, and L. B. Whitbourn, 1989, "Thin capacitive meshes on a dielectric boundary-Theory and experiment," Appl. Opt. 28, 3498-3510.

Degiron, A., H. J. Lezec, W. L. Barnes, and T. W. Ebbesen, 2002, "Effects of hole depth on enhanced light transmission through subwavelength hole arrays," Appl. Phys. Lett. 81, 4327-4329.

Degiron, A., H. J. Lezec, N. Yamamoto, and T. W. Ebbesen, 2004, "Optical transmission properties of a single subwavelength aperture in a real metal," Opt. Commun. 239, 61-66.

Dintinger, J., S. Klein, and T. W. Ebbesen, 2006, "Moleculesurface plasmon interactions in hole arrays: enhanced absorption, refractive index changes, and all-optical switching," Adv. Mater. (Weinheim, Ger.) 18, 1267-1270.

Dintinger, J., I. Robel, P. V. Kamat, C. Genet, and T. W. Ebbesen, 2006, "Terahertz all-optical molecule-plasmon modulation," Adv. Mater. (Weinheim, Ger.) 18, 1645-1648.

Draine, B. T., and P. J. Flatau, 1994, "Discrete-dipole approximation for scattering calculations," J. Opt. Soc. Am. A 11, 1491-1499.

Ebbesen, T. W., H. J. Lezec, H. F. Ghaemi, T. Thio, and P. A. Wolff, 1998, "Extraordinary optical transmission through subwavelength hole arrays," Nature (London) 391, 667-669.

Eggimann, W. H., and R. E. Collin, 1962, "Electromagnetic diffraction by a planar array of circular disks," IRE Trans. Microwave Theory Tech. 10, 528-535.

Ekinci, Y., H. H. Solak, and C. David, 2007, "Extraordinary optical transmission in the ultraviolet region through aluminum hole arrays," Opt. Lett. 32, 172-174.

Elliott, J., I. I. Smolyaninov, N. I. Zheludev, and A. V. Zayats, 2004, "Polarization control of optical transmission of a periodic array of elliptical nanoholes in a metal film," Opt. Lett. 29, 1414-1416.

Falcone, F., T. Lopetegi, M. A. G. Laso, J. D. Baena, J. Bonache, M. Beruete, R. Marqués, F. Martín, and M. Sorolla, 2004, "Babinet principle applied to the design of metasurfaces and metamaterials," Phys. Rev. Lett. 93, 197401.

Fan, W., S. Zhang, B. Minhas, K. J. Malloy, and S. R. J. Brueck, 2005, "Enhanced infrared transmission through subwavelength coaxial metallic arrays," Phys. Rev. Lett. 94, 033902.

Fano, U., 1936, "Some theoretical considerations on anomalous diffraction gratings," Phys. Rev. 50, 573.

Fano, U., 1941, "The theory of anomalous diffraction gratings and of quasi-stationary waves on metallic surfaces (Sommerfeld's waves)," J. Opt. Soc. Am. 31, 213-222.

Fano, U., 1961, "Effects of configuration interaction on intensities and phase shifts," Phys. Rev. 124, 1866-1878.

Farías, D., and K.-H. Rieder, 1998, "Atomic beam diffraction from solid surfaces,” Rep. Prog. Phys. 61, 1575-1664.

Ford, G. W., and W. H. Weber, 1984, "Electromagnetic interactions of molecules with metal surfaces," Phys. Rep. 113, 195-287.

García de Abajo, F. J., 1999, "Interaction of radiation and fast electrons with clusters of dielectrics: a multiple scattering approach," Phys. Rev. Lett. 82, 2776-2779.

García de Abajo, F. J., 2002, "Light transmission through a single cylindrical hole in a metallic film," Opt. Express 10, 1475-1484.

García de Abajo, F. J., R. Gómez-Medina, and J. J. Sáenz, 2005, "Full transmission through perfect-conductor subwavelength hole arrays," Phys. Rev. E 72, 016608.

García de Abajo, F. J., G. Gómez-Santos, L. A. Blanco, A. G. Borisov, and S. V. Shabanov, 2005, "Tunneling mechanism of light transmission through metallic films," Phys. Rev. Lett. 95, 067403.

García de Abajo, F. J., and J. J. Sáenz, 2005, "Electromagnetic surface modes in structured perfect-conductor surfaces," Phys. Rev. Lett. 95, 233901.

García de Abajo, F. J., J. J. Sáenz, I. Campillo, and J. S. Dolado, 2006, "Site and lattice resonances in metallic hole arrays," Opt. Express 14, 7-18.

García-Vidal, F. J., E. Moreno, J. A. Porto, and L. MartínMoreno, 2005, "Transmission of light through a single rectangular hole,” Phys. Rev. Lett. 95, 103901.

García-Vidal, F. J., S. G. Rodrigo, and L. Martín-Moreno, 2006, "Foundations of the composite diffracted evanescent wave model," Nat. Phys. 2, 790-790.

Gay, G., O. Alloschery, B. V. De Lesegno, C. O’Dwyer, J. Weiner, and H. J. Lezec, 2006, "The optical response of nanostructured surfaces and the composite diffracted evanescent wave model," Nat. Phys. 2, 262-267.

Genet, C., and T. W. Ebbesen, 2007, "Light in tiny holes," Nature (London) 445, 39-46.

Genet, C., M. P. van Exter, and J. P. Woerdman, 2003, "Fanotype interpretation of red shifts and red tails in hole array transmission spectra," Opt. Commun. 225, 331-336.

Ghaemi, H. F., T. Thio, D. E. Grupp, T. W. Ebbesen, and H. J. Lezec, 1998, "Surface plasmons enhance optical transmission through subwavelength holes," Phys. Rev. B 58, 6779-6782.

Glasser, M. L., and I. J. Zucker, 1980, in Theoretical Chemistry: Advances and Perspectives, edited by H. Eyring and D. Henderson (Academic, New York), Vol. 5, pp. 67-139.

Gómez-Medina, R., M. Laroche, and J. J. Sáenz, 2006, "Extraordinary optical reflection from sub-wavelength cylinder arrays," Opt. Express 14, 3730-3737.

Gómez-Rivas, J., C. Schotsch, P. Haring Bolivar, and H. Kurz, 2003, "Enhanced transmission of THz radiation through subwavelength holes," Phys. Rev. B 68, 201306(R).

Gordon, R., A. G. Brolo, A. McKinnon, A. Rajora, B. Leathem, and K. L. Kavanagh, 2004, "Strong polarization in the optical transmission through elliptical nanohole arrays," Phys. Rev. Lett. 92, 037401.

Gradshteyn, I. S., and I. M. Ryzhik, 1980, Table of Integrals, Series, and Products (Academic, London).

Greffet, J.-J., R. Carminati, K. Joulain, J.-P. Mulet, S. Mainguy, and Y. Chen, 2002, "Coherent emission of light by thermal sources," Nature (London) 416, 61-64.

Grigorenko, A. N., A. K. Geim, H. F. Gleeson, Y. Zhang, A. A. Firsov, I. Y. Khrushchev, and J. Petrovic, 2005, "Nanofabricated media with negative permeability at visible frequencies," Nature (London) 438, 335-338.

Henke, B. L., E. M. Gullikson, and J. C. Davis, 1993, "X-ray interactions: photoabsorption, scattering, transmission, and reflection at $E=50-30,000 \mathrm{eV}, Z=1-92$," At. Data Nucl. Data Tables 54, 181-342.

Hibbins, A. P., B. R. Evans, and J. R. Sambles, 2005, "Experimental verification of designer surface plasmons," Science 308, 670-672.

Hibbins, A. P., M. J. Lockyear, I. R. Hooper, and J. R. 
Sambles, 2006, "Waveguide arrays as plasmonic metamaterials: transmission below cutoff," Phys. Rev. Lett. 96, 073904.

Hicks, E. M., S. Zou, G. C. Schatz, K. G. Spears, R. P. Van Duyne, L. Gunnarsson, T. Rindzevicius, B. Kasemo, and M. Käll, 2005, "Controlling plasmon line shapes through diffractive coupling in linear arrays of cylindrical nanoparticles fabricated by electron beam lithography," Nano Lett. 5, 1065 1070.

Hillenbrand, R., T. Taubner, and F. Keilmann, 2002, "Phononenhanced light-matter interaction at the nanometer scale," Nature (London) 418, 159-162.

Huang, F. M., N. Zheludev, Y. Chen, and F. J. García de Abajo, 2007, "Focusing of light by a nano-hole array," Appl. Phys. Lett. 90, 091119.

Hutley, M. C., and D. Maystre, 1976, "The total absorption of light by a diffraction grating," Opt. Commun. 19, 431-436.

Jackson, J. D., 1999, Classical Electrodynamics (Wiley, New York).

James, G. L., 1977, "Radiation properties of 90 degrees conical horns," Electron. Lett. 13, 293-294.

Janke, C., J. Gómez Rivas, P. Haring Bolivar, and H. Kurz, 2005, "All-optical switching of the transmission of electromagnetic radiation through subwavelength apertures," Opt. Lett. 30, 2357-2359.

Joannopoulos, J. D., P. R. Villeneuve, and S. H. Fan, 1997, "Photonic crystals: putting a new twist on light," Nature (London) 386, 143-149.

Johnson, P. B., and R. W. Christy, 1972, "Optical constants of the noble metals," Phys. Rev. B 6, 4370-4379.

Jones, R. C., 1945, "A generalization of the dielectric ellipsoid problem,” Phys. Rev. 68, 93-96.

Kambe, K., 1968, "Theory of low-energy electron diffraction 2. Cellular method for complex monolayers and multilayers," $\mathrm{Z}$. Naturforsch. A 23, 1280-1294.

Kelf, T. A., Y. Sugawara, J. J. Baumberg, M. Abdelsalam, and P. N. Bartlett, 2005, "Plasmonic band gaps and trapped plasmons on nanostructured metal surfaces," Phys. Rev. Lett. 95, 116802.

Kelf, T. A., Y. Sugawara, R. M. Cole, J. J. Baumberg, M. E. Abdelsalam, S. Cintra, S. Mahajan, A. E. Russell, and P. N. Bartlett, 2006, "Localized and delocalized plasmons in metallic nanovoids," Phys. Rev. B 74, 245415.

Kitson, S. C., W. L. Barnes, and J. R. Sambles, 1996, "Full photonic band gap for surface modes in the visible," Phys. Rev. Lett. 77, 2670-2673.

Klein Koerkamp, K. J., S. Enoch, F. B. Segerink, N. F. van Hulst, and L. Kuipers, 2004, "Strong influence of hole shape on extraordinary transmission through periodic arrays of subwavelength holes," Phys. Rev. Lett. 92, 183901.

Krasavin, A. V., A. S. Schwanecke, N. I. Zheludev, M. Reichelt, T. Stroucken, S. W. Koch, and E. M. Wright, 2005, "Polarization conversion and focusing of light propagating through a small chiral hole in a metallic screen," Appl. Phys. Lett. 86, 201105.

Krenn, J. R., A. Dereux, J. C. Weeber, E. Bourillot, Y. Lacroute, J. P. Goudonnet, G. Schider, W. Gotschy, A. Leitner, F. R. Aussenegg, and C. Girard, 1999, "Squeezing the optical near-field zone by plasmon coupling of metallic nanoparticles," Phys. Rev. Lett. 82, 2590-2593.

Krishnan, A., T. Thio, T. J. Kim, H. J. Lezec, T. W. Ebbesen, P. A. Wolff, J. Pendry, L. Martín-Moreno, and F. J. GarcíaVidal, 2001, "Evanescently coupled resonance in surface plasmon enhanced transmission," Opt. Commun. 200, 1-7.
Lalanne, P., and J. P. Hugonin, 2006, "Interaction between optical nano-objects at metallo-dielectric interfaces," Nat. Phys. 2, 551-556.

Laroche, M., S. Albaladejo, R. Gómez-Medina, and J. J. Sáenz, 2006, "Tuning the optical response of nanocylinder arrays: an analytical study," Phys. Rev. B 74, 245422.

Lezec, H. J., A. Degiron, E. Devaux, R. A. Linke, L. MartínMoreno, F. J. García-Vidal, and T. W. Ebbesen, 2002, "Beaming light from a subwavelength aperture," Science 297, 820822 .

Lezec, H. J., and T. Thio, 2004, "Diffracted evanescent wave model for enhanced and suppressed optical transmission through subwavelength hole arrays," Opt. Express 12, 36293651.

Liz-Marzán, L. M., 2006, "Tailoring surface plasmon through the morphology and assembly of metal nanoparticles," Langmuir 22, 32-41.

Loewen, E. G., W. R. McKinney, and R. McPhedran, 1984, in Application, Theory, and Fabrication of Periodic Structures, edited by J. M. Lerner (SPIE, Bellingham, WA), Vol. 503, pp. 187-197.

López, C., 2003, "Materials aspects of photonic crystals," Adv. Mater. (Weinheim, Ger.) 15, 1679-1704.

Lord Rayleigh, 1907, "Note on the remarkable case of diffraction spectra described by Prof. Wood," Philos. Mag. 14, 6065.

Maier, S. A., M. L. Brongersma, P. G. Kik, S. Meltzer, A. A. G. Requicha, and H. A. Atwater, 2001, "Plasmonics-a route to nanoscale optical devices," Adv. Mater. (Weinheim, Ger.) 13, 1501-1505.

Maier, S. A., P. G. Kik, H. A. Atwater, S. Meltzer, E. Harel, B. E. Koel, and A. A. G. Requicha, 2003, "Local detection of electromagnetic energy transport below the diffraction limit in metal nanoparticle plasmon waveguides," Nat. Mater. 2, 229-232.

Marquier, F., J.-J. Greffet, S. Collin, F. Pardo, and J. L. Pelouard, 2005, "Resonant transmission through a metallic film due to coupled modes," Opt. Express 13, 70-76.

Martín-Moreno, L., F. J. García-Vidal, H. J. Lezec, K. M. Pellerin, T. Thio, J. B. Pendry, and T. W. Ebbesen, 2001, "Theory of extraordinary optical transmission through subwavelength hole arrays," Phys. Rev. Lett. 86, 1114-1117.

Martínez-Sala, R., J. Sancho, J. V. Sánchez, V. Gómez, J. Llinares, and F. Meseguer, 1995, "Sound attenuation by sculpture," Nature (London) 378, 241-241.

Matsui, T., A. Agrawal, A. Nahata, and Z. V. Vardeny, 2007, "Transmission resonances through aperiodic arrays of subwavelength apertures," Nature (London) 446, 517-521.

Maystre, D., 1972, "Sur la diffraction d'une onde plane par un reseau metallique de conductivite finie," Opt. Commun. 6, $50-54$.

Maystre, D., 1980, in Electromagnetic Theory of Gratings, edited by R. Petit (Springer-Verlag, Berlin), pp. 63-100.

Maystre, D., 1984, "Rigorous vector theories of diffraction gratings," Prog. Opt. 21, 1-67.

Maystre, D., 1993, Diffraction Gratings, SPIE Milestones Series, Vol. MS 83 (SPIE, Bellingham, WA).

McPhedran, R. C., G. H. Derrick, and L. C. Botten, 1980, in Electromagnetic Theory of Gratings, edited by R. Petit (Springer-Verlag, Berlin), pp. 227-276.

McPhedran, R. C., and D. Maystre, 1974, "A detailed theoretical study of the anomalies of a sinusoidal diffraction grating," Opt. Acta 21, 413-421. 
Mie, G., 1908, "Beiträge zur Optik trüber Medien, speziell kolloidaler Metallösungen," Ann. Phys. 25, 377-445.

Milton, G. W., 2002, The Theory of Composites (Cambridge University Press, Cambridge, England).

Mittra, R., C. H. Chan, and T. Cwik, 1988, "Techniques for analyzing frequency selective surfaces-A review," Proc. IEEE 76, 1593-1615.

Miyamaru, F., and M. Hangyo, 2004, "Finite size effect of transmission property for metal hole arrays in subterahertz region," Appl. Phys. Lett. 84, 2742-2744.

Nomura, W., M. Ohtsu, and T. Yatsui, 2005, "Nanodot coupler with a surface plasmon polariton condenser for optical far/ near-field conversion," Appl. Phys. Lett. 86, 181108.

Nordlander, P., C. Oubre, E. Prodan, K. Li, and M. I. Stockman, 2004, "Plasmon hybridizaton in nanoparticle dimers," Nano Lett. 4, 899-903.

Obermüller, C., and K. Karrai, 1995, "Far field characterization of diffracting circular apertures," Appl. Phys. Lett. 67, 3408-3410.

Ozbay, E., 2006, "Plasmonics: merging photonics and electronics at nanoscale dimensions," Science 311, 189-193.

Palik, E. D., 1985, Handbook of Optical Constants of Solids (Academic, New York).

Papasimakis, N., V. A. Fedotov, A. S. Schwanecke, N. I. Zheludev, and F. J. García de Abajo, 2007, "Enhanced microwave transmission through quasicrystal hole arrays," Appl. Phys. Lett. 91, 081503.

Pendry, J. B., 1974, Low Energy Electron Diffraction (Academic, London).

Pendry, J. B., L. Martín-Moreno, and F. J. García-Vidal, 2004, "Mimicking surface plasmons with structured surfaces," Science 305, 847-848.

Pettit, R. B., J. Silcox, and R. Vincent, 1975, "Measurement of surface-plasmon dispersion in oxidized aluminum films," Phys. Rev. B 11, 3116-3123.

Popov, E., N. Bonod, M. Nevière, H. Rigneault, P.-F. Lenne, and P. Chaumet, 2005, "Surface plasmon excitation on a single subwavelength hole in a metallic sheet," Appl. Opt. 44, 2332-2337.

Popov, E., M. Nevière, S. Enoch, and R. Reinisch, 2000, "Theory of light transmission through subwavelength periodic hole arrays,” Phys. Rev. B 62, 16100-16108.

Porto, J. A., F. J. García-Vidal, and J. B. Pendry, 1999, “Transmission resonances on metallic gratings with very narrow slits," Phys. Rev. Lett. 83, 2845-2848.

Powell, C. J., and J. B. Swan, 1959, "Origin of the characteristic electron energy losses in aluminum," Phys. Rev. 115, 869875.

Przybilla, F., A. Degiron, J.-Y. Laluet, C. Genet, and T. W. Ebbesen, 2006, "Optical transmission in perforated noble and transition metal films," J. Opt. A, Pure Appl. Opt. 8, 458-463.

Przybilla, F., C. Genet, and T. W. Ebbesen, 2006, "Enhanced transmission through Penrose subwavelength hole arrays," Appl. Phys. Lett. 89, 121115.

Purcell, E. M., and C. R. Pennypacker, 1973, "Scattering and absorption of light by nonspherical dielectric grains," Astrophys. J. 186, 705-714.

Quinten, M., A. Leitner, J. R. Krenn, and F. R. Aussenegg, 1998, "Electromagnetic energy transport via linear chains of silver nanoparticles," Opt. Lett. 23, 1331-1333.

Raether, H., 1988, Surface Plasmons on Smooth and Rough Surfaces and on Gratings, Springer Tracts in Modern Physics Vol. 111 (Springer-Verlag, Berlin).
Reif, F., 1965, Fundamentals of Statistical and Thermal Physics (McGraw-Hill, New York).

Rindzevicius, T., Y. Alaverdyan, B. Sepulveda, T. Pakizeh, M. Käll, R. Hillenbrand, J. Aizpurua, and F. J. García de Abajo, 2007, "Nanohole plasmons in optically thin gold films," J. Phys. Chem. C. 111, 1207-1212.

Ritchie, R. H., 1957, "Plasma losses by fast electrons in thin films," Phys. Rev. 106, 874-881.

Ritchie, R. H., E. T. Arakawa, J. J. Cowan, and R. N. Hamm, 1968, "Surface-plasmon resonance effect in grating diffraction," Phys. Rev. Lett. 21, 1530-1533.

Roberts, A., 1987, "Electromagnetic theory of diffraction by a circular aperture in a thick, perfectly conducting screen," J. Opt. Soc. Am. A 4, 1970-1983.

Roberts, A., and R. C. McPhedran, 1988, "Bandpass grids with annular apertures," IEEE Trans. Antennas Propag. 36, 607611.

Romero, I., J. Aizpurua, G. W. Bryant, and F. J. García de Abajo, 2006, "Plasmons in nearly touching metallic nanoparticles: singular response in the limit of touching dimers," Opt. Express 14, 9988-9999.

Ruan, Z., and M. Qiu, 2006, "Enhanced transmission through periodic arrays of subwavelength holes: The role of localized waveguide resonances," Phys. Rev. Lett. 96, 233901.

Salomon, L., F. Grillot, A. V. Zayats, and F. de Fornel, 2001, "Near-field distribution of optical transmission of periodic subwavelength holes in a metal film," Phys. Rev. Lett. 86, 1110-1113.

Sarid, D., 1981, "Long-range surface-plasma waves on very thin metal films," Phys. Rev. Lett. 47, 1927-1930.

Sarrazin, M., J.-P. Vigneron, and J.-M. Vigoureux, 2003, "Role of Wood anomalies in optical properties of thin metallic films with a bidimensional array of subwavelength holes," Phys. Rev. B 67, 085415.

Schröter, U., and D. Heitmann, 1998, "Surface-plasmonenhanced transmission through metallic gratings," Phys. Rev. B 58, 15419-15421.

Schuster, S. C., R. V. Swanson, L. A. Alex, R. B. Bourret, and M. I. Simon, 1993, "Assembly and function of a quaternary signal-transduction complex monitored by surface-plasmon resonance," Nature (London) 365, 343-347.

Schwanecke, A. S., N. Papasimakis, V. A. Fedotov, F. Huang, Y. Chen, F. J. García de Abajo, and N. I. Zheludev, 2006, nanophotonics topical meeting NANO at IPRA/NANO OSA Collocated Topical Meetings, Uncasville, CT (unpublished).

Selcuk, S., K. Woo, D. B. Tanner, A. F. Hebard, A. G. Borisov, and S. V. Shabanov, 2006, "Trapped electromagnetic modes and scaling in the transmittance of perforated metal films," Phys. Rev. Lett. 97, 067403.

Smith, D. R., J. B. Pendry, and M. C. K. Wiltshire, 2004, "Metamaterials and negative refractive index," Science 305, 788-792.

Smolyaninov, I. I., A. V. Zayats, A. Stanishevsky, and C. C. Davis, 2002, "Optical control of photon tunneling through an array of nanometer-scale cylindrical channels," Phys. Rev. B 66, 205414.

Stefanou, N., V. Yannopapas, and A. Modinos, 1998, "Heterostructures of photonic crystals: frequency bands and transmission coefficients," Comput. Phys. Commun. 113, 49-77.

Stefanou, N., V. Yannopapas, and A. Modinos, 2000, "MULTEM 2: a new version of the program for transmission and band-structure calculations of photonic crystals," Comput. Phys. Commun. 132, 189-196. 
Stewart, J. E., and W. S. Gallaway, 1962, "Diffraction anomalies in grating spectrophotometers," Appl. Opt. 1, 421-429.

Stuart, H. R., and D. G. Hall, 1998, "Enhanced dipole-dipole interaction between elementary radiators near a surface," Phys. Rev. Lett. 80, 5663-5666.

Sun, M., J. Tian, Z.-Y. Li, B.-Y. Cheng, D.-Z. Zhang, A.-Z. Jin, and H.-F. Yang, 2006, "The role of periodicity in enhanced transmission through subwavelength hole arrays," Chin. Phys. Lett. 23, 486-488.

Takakura, Y., 2001, "Optical resonance in a narrow slit in a thick metallic screen," Phys. Rev. Lett. 86, 5601-5603.

Talbot, H. F., 1836, "Facts relating to optical science, No. IV," Philos. Mag. 9, 401-407.

Teperik, T. V., V. V. Popov, and F. J. García de Abajo, 2005, "Void plasmons and total absorption of light in nanoporous metallic films," Phys. Rev. B 71, 085408.

Teperik, T. V., V. V. Popov, F. J. García de Abajo, M. Abdelsalam, P. N. Barlett, T. A. Kelf, Y. Sugawara, and J. J. Baumberg, 2006, "Strong coupling of light to flat metals via a buried nanovoid lattice: the interplay of localized and free plasmons," Opt. Express 14, 1965-1972.

Teperik, T. V., V. V. Popov, F. J. García de Abajo, T. A. Kelf, Y. Sugawara, J. J. Baumberg, M. Abdelsalam, and P. N. Bartlett, 2006, "Mie plasmon enhanced diffraction of light from nanoporous metal surfaces,” Opt. Express 14, 11964-11971.

Treacy, M. M. J., 1999, "Dynamical diffraction in metallic optical gratings," Appl. Phys. Lett. 75, 606-608.

Treacy, M. M. J., 2002, "Dynamical diffraction explanation of the anomalous transmission of light through metallic gratings," Phys. Rev. B 66, 195105.

Ulrich, R., 1967, "Far-infrared properties of metallic mesh and its complementary structure," Infrared Phys. 7, 37-55.

Ulrich, R., and M. Tacke, 1972, "Submillimeter waveguiding on periodic metal structure," Appl. Phys. Lett. 22, 251-253.

van Coevorden, D. V., R. Sprik, A. Tip, and A. Lagendijk, 1996, "Photonic band structure of atomic lattices," Phys. Rev. Lett. 77, 2412-2415.

van de Hulst, H. C., 1981, Light Scattering by Small Particles (Dover, New York).

van der Molen, K. L., K. J. Klein Koerkamp, S. Enoch, F. B. Segerink, N. F. van Hulst, and L. Kuipers, 2005, "Role of shape and localized resonances in extraordinary transmission through periodic arrays of subwavelength holes: experiment and theory," Phys. Rev. B 72, 045421.

Vincent, R., and J. Silcox, 1973, "Dispersion of radiative sur- face plasmons in aluminum films by electron scattering," Phys. Rev. Lett. 31, 1487-1490.

Wannemacher, R., 2001, "Plasmon-supported transmission of light through nanometric holes in metallic thin films," Opt. Commun. 195, 107-118.

Webb, K. J., and J. Li, 2006, "Analysis of transmission through small apertures in conducting films," Phys. Rev. B 73, 033401. Weber, W. H., and G. W. Ford, 2004, "Propagation of optical excitations by dipolar interactions in metal nanoparticle chains," Phys. Rev. B 70, 125429.

Weyl, H., 1919, "Ausbreitung elektromagnetisher Wellen ueber einem. ebenen Leiter," Ann. Phys. 60, 481-500.

Wood, R. W., 1902, "On a remarkable case of uneven distribution of light in a diffraction grating spectrum," Philos. Mag. 4, 396-402.

Wood, R. W., 1912, "Diffraction gratings with controlled groove form and abnormal distribution of intensity," Philos. Mag. 23, 310-317.

Wood, R. W., 1935, “Anomalous diffraction gratings," Phys. Rev. 48, 928-936.

Yang, F., and J. R. Sambles, 2002, "Resonant transmission of microwaves through a narrow metallic slit," Phys. Rev. Lett. 89, 063901.

Yang, F., J. R. Sambles, and G. W. Bradberry, 1990, "Longrange coupled surface exciton polaritons," Phys. Rev. Lett. 64, 559-562.

Yin, L., V. K. Vlasko-Vlasov, A. Rydh, J. Pearson, U. Welp, S.-H. Chang, S. K. Gray, G. C. Schatz, D. B. Brown, and C. W. Kimball, 2004, "Surface plasmons at single nanoholes in Au films," Appl. Phys. Lett. 85, 467-469.

Zenneck, J., 1907, "Uber die Fortpflanzung ebener elektromagnetischer Wellen langs einer ebenen Leiterflache und ihre Beziehung zur drahtlosen Telegraphie," Ann. Phys. 23, 846-866.

Zia, R., J. A. Schuller, A. Chandran, and M. L. Brongersma, 2006, "Plasmonics: the next chip-scale technology," Mater. Today 9, 20-27.

Zou, S., N. Janel, and G. C. Schatz, 2004, "Silver nanoparticle array structures that produce remarkably narrow plasmon lineshapes," J. Chem. Phys. 120, 10871-10875.

Zou, S., and G. C. Schatz, 2004, "Narrow plasmonic/photonic extinction and scattering line shapes for one and two dimensional silver nanoparticle arrays," J. Chem. Phys. 121, 1260612612. 